\title{
Robust Control and Hot Spots in Spatiotemporal Economic Systems *
}

\author{
W. A. Brock ${ }^{1 \dagger}$, A. Xepapadeas ${ }^{2}$ and A. N. Yannacopoulos ${ }^{3}$, \\ (1) Department of Economics, \\ University of Wisconsin, Madisson, wbrock@ssc.wisc.edu \\ (2) Department of International and European Studies, \\ Athens University of Economics and Business, xepapad@aueb.gr, \\ (3) Department of Statistics, \\ Athens University of Economics and Business, ayannaco@aueb.gr
}

July 26, 2012

\begin{abstract}
We formulate stochastic robust optimal control problems, motivated by applications arising in interconnected economic systems, or spatially extended economies. We study in detail linear quadratic problems and nonlinear problems. We derive optimal robust controls and identify conditions under which concerns about model misspecification at specific site(s) could cause regulation to break down, to be very costly, or to induce pattern formation and spatial clustering. We call sites associated with these phenomena hot spots. We also provide an application of our methods by studying optimal robust control and the potential break down of regulation, due to hot spots, in a model where utility for in situ consumption is distance dependent.

Keywords: stochastic control, robust control, Riccati equation, Hot spot formation, Pattern formation, Hamilton-Jacobi Bellman equation, Distance dependent utility.
\end{abstract}

JEL classification: C6, R12, Q26.

${ }^{*}$ This research has been co-financed by the European Union (European Social Fund - ESF) and Greek national funds through the Operational Program "Education and Lifelong Learning" of the National Strategic Reference Framework (NSRF) - Research Funding Program: "Thalis - Athens University of Economics and Business - Optimal Management of Dynamical Systems of the Economy and the Environment: The Use of Complex Adaptive Systems".

${ }^{\dagger} \mathrm{W}$. Brock is grateful for financial and scientific support received from The Center for Robust Decision Making on Climate and Energy Policy (RDCEP) which is funded by a grant from the National Science Foundation (NSF) through the Decision Making Under Uncertainty (DMUU) program. He is also grateful to the Vilas Trust for financial support. None of the above are responsible for any errors, opinions, or shortcomings in this article 


\section{Introduction}

Decision making when the decision making agent has concerns about possible deviations of the model used in the decision making process from the model specified, have been associated with the concept of robustness. Whittle (1996) characterizes a rule as robust if it continues to behave well even if the model deviates from a specified or a benchmark model and points out that optimality should be supplemented by robustness. Thus the desire for robustness emerges when the decision maker regards her model not as the correct one but as an approximation of the correct one, or to put it differently, when the decision maker has concerns about possible misspecifications of the reference model and wants to incorporate these concerns into the decision-making rules. Robust control problems have been traditionally analyzed in the context of risk sensitive linear quadratic Gaussian (LEQG) models and the $\mathrm{H}^{\infty}$ models (e.g. Başar and Bernhard (2008), Whittle (1996)). The $\mathrm{H}^{\infty}$ criterion implies decision making for protection against that 'worst case' and is related to a minimax approach.

More recently Hansen and Sargent (Hansen and Sargent (2001)) interpreted concerns about model misspecification in economics as a situation where a decision maker or a regulator distrusts her model and wants good decisions over a cloud of models that surrounds the regulator's approximating or benchmark model, which are difficult to distinguish with finite data sets. ${ }^{1}$. Then they obtain robust decisions rules by introducing a fictitious 'adversarial agent' which we will refer to as Nature. Nature promotes robust decision rules by forcing the regulator, who seeks to maximize (minimize) an objective, to explore the fragility of decision rules to departures from the benchmark model. A robust decision rule means that lower bounds to the rule's performance are determined by Nature - the adversarial agent - who acts as a minimizing (maximizing) agent when constructing these lower bounds. Hansen and Sargent (Hansen et al. (2006)) show that robust control theory can be interpreted as a recursive version of max-min expected utility theory (Gilboa and Schmeidler (1989)). In this context the decision maker cannot or does not formulate a single probability model and maximizes expected utility assuming the probability weights are chosen by Nature, the adversarial agent.

Robust control methods have been extensively used to study dynamic models, but no extension has been undertaken, as far as we know, to models that evolve both in time and space. Decision making when the spatial dimension of underlying problem is explicitly taken into account and the decision maker or a regulator seeks to determine spatially dependent rules is attracting increasing interest in economics. The spatial dimension has been brought into the picture through new economic geography models (e.g., Krugman (1996), Boucekkine et al. (2009), Desmet and Rossi-Hansberg (2010)), but also through models of resource management (e.g. Sanchirico and Wilen (1999), Wilen (2007), Smith et al. (2009), Brock and Xepapadeas $(2008,2010)$ ). In fields like biology or automatic control, systems with spatially distributed parameter aspects in the dynamics have been used to study pattern formation on biological agents (e.g., Murray (2003)), the control of infinite platoons of vehicles over time (e.g., Bamieh et al. (2002), Curtain et al. (2008)), or groundwater management (e.g., Leizarowitz (2008)).

The new element that the spatial dimension brings into robust control is that concerns about model misspecification refer now to the benchmark or reference model that describes the spatiotemporal dynamics of each specific site. If potential deviations from the specified model differ from site to site then concerns for one site might affect the robust rules for other sites, given the interrelations among different sites through short or long range spatial effects.

Thus the regulator should design the robust rules not only with respect to the spatial characteristics of the problem in a specific location but also with respect to the degree to which the regulator distrusts her model across locations. This means that if concerns about the benchmark model in a given site differ from concerns in other sites, a spatially dependent robust rule should capture these differences. This observation allows us to formally identify, for the first time to our knowledge in economics, spatial hot spots - which are sites where robust control breaks down - or sites where robust control is very costly as a function of the degree of the regulator's concerns about model misspecification across all

\footnotetext{
${ }^{1}$ see also Salmon (2002); Hansen et al. (2006); JET (2006), Hansen and Sargent (2008).
} 
sites. We are also able to identify spatial hot spots where the need to apply robust control induces spatial agglomerations and breaks down spatial symmetry. From the theory point of view this is, as far as we know, a new source for generating spatial patterns as compared to the classic Turing diffusion induced instability (Turing (1952)) and the more recently identified optimal diffusion or spatial-spillover-induced instabilities (Brock and Xepapadeas $(2008,2010)$ ). Thus hot spots are specific sites where uncertainties in these sites are such that when concerns about local misspecifications are incorporated into the decision rules for the entire spatial domain, the global rule could break down, could be very costly or could induce spatial clustering.

This result regarding robust control in spatiotemporal systems brings up another point which could be associated with applied policy design and regulation. It has been argued recently (e.g., Haldane (2009)) that increased interconnectedness among networks has made various networks, such as ecological networks, power grid networks, transportation networks, financial networks more unstable. This interconnectedness and the potential instabilities induced can be associated with the hot spots introduced by our model and the impact of local properties on global regulation. ${ }^{2}$

In the rest of the paper we formalize local concerns with the help of local entropy constraints and we derive robust control rules, for a general linear quadratic model and a special case of this model where translation invariance allows the derivation of closed form results, as well a general nonlinear model. We also show how robust control can be applied using linear quadratic approximations. Finally we provide an economic application where utility is spatially dependent and consumers consume in situ ecosystem services by traveling to locations. We provide robust decision rules for an optimal linear regulation problem where the objectives is to determine the optimal supply of services in each site so that equilibrium local fees are determined. We show how misspecification concerns about local resource dynamics could break down regulation and induce spatial patterns.

\section{Modeling a spatial economy under uncertainty}

\subsection{The controlled state equation}

Consider the economy as being located on a discrete finite lattice $\mathfrak{L}$, e.g. $\mathfrak{L}=\left(\mathbb{Z}_{N}\right)^{d}$. By the term "economy" at this point we consider a collection of state variables $x=\left\{x_{n}\right\}, n \in \mathfrak{L}$. For fixed $n, x_{n} \in \mathbb{R}$ and corresponds to the state of the economy at lattice site $n$. We therefore consider the state variable $x$ as taking values on a (finite dimensional) sequence space. To keep our discussion within a Hilbert space setting we choose to work with the sequence space $\ell^{2}:=\ell^{2}\left(\mathbb{Z}_{N}\right)=\left\{\left\{x_{n}\right\}, \sum_{n \in \mathbb{Z}_{N}} x_{n}^{2}<\infty\right\}^{3}$. This space is a Hilbert space with a norm derivable from the inner product $\langle x, y\rangle=\sum_{n \in \mathbb{Z}_{N}} x_{n} y_{n}$ and is in fact equivalent to $\mathbb{R}^{N}$. Given this economy we consider a social planning problem modelled as an optimal linear regulator problem (e.g.Ljungqvist and Sargent (2004)). The optimal linear regulator problem refers to the optimization of a quadratic objective defined over the whole lattice by exerting on each lattice site a control $u_{n} \in \mathbb{R}$ where the control for the whole economy is described as a sequence $u=\left\{u_{n}\right\}, n \in \mathbb{Z}_{N}$ such that $u \in \ell^{2}\left(\mathbb{Z}_{N}\right)=\mathbb{R}^{N 4}$. From now on to simplify notation we will simply denote the state space for the economy by $\mathbb{R}^{N}$.

\footnotetext{
${ }^{2}$ Although we choose to interpret the characteristics associated with the distributed parameter aspect as physical space, the notion of "space" does not have to be physical. It can be used to model characteristics that are associated with economic, sociological, cultural or other factors. Since the notion of "space" may be broadly interpreted, this suggests that our methods can be used for the analysis of a wide range of problems.

${ }^{3}$ This choice is for simplicity of presentation. Most of the arguments and results presented here can be extended to infinite dimensional systems, admittedly with considerable technical effort employing techniques beyond the scope of the present paper, or when explicitly stated so with a weighted version of this space.

${ }^{4}$ The generalization to vector valued state and control variables $x^{n} \in \mathbb{R}^{d_{1}}$ and $u_{n} \in \mathbb{R}^{d_{2}}$ on each site $n \in \mathbb{Z}$ requires the use of the sequence spaces $\ell^{2}\left(\mathbb{R}^{d_{i}}\right), i=1,2$ rather than $\left.\ell^{2}:=\ell^{2}(\mathbb{R})\right)$ and is straightforward. Furthermore, the generalization for infinite dimensional lattices is feasible, but becomes technical from the mathematical point of view and is beyond the scope of the present paper.
} 
The economy evolves in time and this is modelled by considering the state of the economy as described by a function $\check{x}: I \rightarrow \mathbb{R}^{N}$ such that $\check{x}(t)=\left\{x_{n}(t)\right\}, n \in \mathfrak{L}$, where $x_{n}(t)$ is the state of the system at site $n$ at time $t$. To ease notation we will use the notation $x$ for this function and similarly $u$ for the control exerted on the system. In this paper, we are interested in an infinite horizon economy and thus we assume $I=\mathbb{R}_{+}$. The evolution of the state of the economy in time is subject to statistical fluctuations (noise), which is introduced into the model via stochastic factors (sources) ${ }^{5}$, modelled in terms of a stochastic process $w=\left\{w_{n}\right\}, n \in \mathbb{Z}_{N}$, which is considered as a vector valued Wiener process on a suitable filtered probability space $\left(\Omega,\left\{\mathcal{F}_{t}\right\}_{t \in \mathbb{R}_{+}}, \mathcal{F}, P\right)$ (see e.g., Karatzas and Shreve (1991)). The introduction of noise turns the state of the system for a fixed time $t$ into an $\mathbb{R}^{N}$-valued random variable, thus the state of the system can be described as an $\mathbb{R}^{N}$-valued stochastic process. We assume that this stochastic process is the solution of a stochastic differential equation of the form

$$
d x_{n}=\left(\sum_{m} a_{n m} x_{m}+\sum_{m} b_{n m} u_{m}\right) d t+\sum_{m} c_{n m} d w_{m}, \quad n \in \mathbb{Z}_{N}
$$

where the last term, describing the fluctuations of the state due to the stochasticity, is understood in the sense of the Itō theory of stochastic integration. In compact form this can be expressed as

$$
d x=(\mathrm{A} x+\mathrm{B} u) d t+\mathrm{C} d w
$$

where $\mathrm{A}, \mathrm{B}, \mathrm{C}: \mathbb{R}^{N} \rightarrow \mathbb{R}^{N}$ are linear operators, representable by finite matrices with elements $a_{n m}, b_{n m}$, $c_{n m}$, respectively. The state equation (1) is an Ornstein-Uhlenbeck equation on the finite dimensional Hilbert space $\ell^{2}\left(\mathbb{Z}_{N}\right)=\mathbb{R}^{N}$.

At this point we make some comments concerning the economic intuition behind the state equation (1). Our model is a "spatial" economy where it is considered that the state of the economy at point $m$ has an effect at the state of the economy at point $n$. This effect is quantified through an influence "kernel" (or rather a discretized version of an influence kernel) which can be represented in terms of a matrix $\mathrm{A}=\left(a_{n m}\right)$. The entry $a_{n m}$ provides a measure of the influence of the state of the system at point $m$ to the state of the system at point $n$. Network effects knowledge spillovers can be modelled for example through a proper choice of A. For instance, if the economies do not interact at all then $\mathrm{A}=a_{n m}=\delta_{n, m}$ where $\delta_{n, m}$ is the Kronecker delta. If only next neighborhood effects are possible then $a_{n m}$ is non-zero only if $m$ is a neighbor of $n$. Such an example is the discrete Laplacian. Similarly, the controls at different point of the lattice $u_{m}$ are assumed to have an effect at the state of the system at site $n$, through the term $\sum_{m} b_{n m} u_{m}$. For example in a model of a spatial fishery, fishing effort at a given site may affect fish biomass at another sites through biomass movements. A similar interpretation for this term holds as for the term $\sum_{m} a_{n m} x_{m}$. We will identify the matrices $\mathrm{A}=\left(a_{n m}\right)$ and $\mathrm{B}=\left(b_{n m}\right)$ with operators denoted by the same symbol, acting from $\mathbb{R}^{N} \rightarrow \mathbb{R}^{N}$.

Finally, the interpretation of the third term $\sum_{m} c_{n m} d w_{m}$ is a term that tells us how the uncertainty at site $m$ is affecting the uncertainty concerning the state of the system at site $n$. The matrix $C=\left(c_{n m}\right)$ can be thought of as the spatial autocorrelation operator for the system.

\subsection{Model uncertainty}

Assume now that there is some uncertainty concerning the "true" statistical distribution of the state of the system. This corresponds to a family of probability measures $\mathcal{Q}$ such that each $Q \in \mathcal{Q}$ corresponds to an alternative stochastic model (scenario) concerning the state of the system. Considering for

\footnotetext{
${ }^{5}$ There is uncertainty concerning the economy which is represented in terms of the vector valued stochastic process $w$. These common factors affect the state of the economy $x$ at the different sites. Each factor has a different effect on the state of the economy on each particular site; this will be modelled by a suitable correlation matrix. It is not of course necessary that the number of factors is the same as the number of sites in the system however, without loss of generality we will make this assumption and assume that there is one factor or source of uncertainty related to each site. This assumption can be easily relaxed.
} 
the time being finite horizon $T$, we restrict ourselves to measures which are equivalent with $P$ (i.e. having the same null sets) such that the Radon-Nikodym derivatives $d Q / d P$ are defined through an exponential martingale of the type employed in Girsanov's theorem,

$$
\left.\frac{d Q}{d P}\right|_{\mathcal{F}_{T}}=\exp \left(\int_{0}^{T} \sum_{n} v_{n}(t) d w_{n}(t)-\frac{1}{2} \int_{0}^{T} \sum_{n} v_{n}^{2}(t) d t\right),
$$

where $v=\left\{v_{n}\right\}, n \in \mathbb{Z}_{N}$ is an $\mathbb{R}^{N}$-valued stochastic process which is measurable with respect to the filtration $\left\{\mathcal{F}_{t}\right\}$ satisfying the Novikov condition $\exp \left(\int_{0}^{T} \sum_{n} v_{n}^{2}(t) d t\right)<\infty$. If the process $v$ can be chosen so that this condition is true for all $T$, then the result in the infinite horizon limit follows by appropriately passing to the limit $T \rightarrow \infty$. Furthermore, the same theorem guarantees that $\bar{w}_{n}(t)=w_{n}(t)-\int_{0}^{t} v_{n}(s) d s$ is a $Q$-Brownian motion for all $n \in \mathbb{N}$, where the drift term $v_{n}$ may be considered as a measure of the model misspecification at lattice site $n$. Thus, Girsanov's theorem (see e.g. Karatzas and Shreve (1991)) shows that the adoption of the family $\mathcal{Q}$ of alternative measures concerning the state of the system, leads to a family of differential equations for the state variable

$$
d x_{n}=\left(\sum_{m} a_{n m} x_{m}+\sum_{m} b_{n m} u_{m}+\sum_{m} c_{n m} v_{m}\right) d t+\sum_{m} c_{n m} d \bar{w}_{m}, \quad n \in \mathbb{Z}_{N}
$$

The state variables $x=\left\{x_{n}\right\}$ depend on the choice of $u=\left\{u_{n}\right\}$ and $v=\left\{v_{n}\right\}$ therefore, $x=x^{u, v}$, however we choose to avoid this notation for simplicity. We therefore tacitly assume that $x$ indicates the state of the system when the measure $Q$ corresponding to the "information drift" $v=\left\{v_{n}\right\}$ and the control procedure $u=\left\{u_{n}\right\}$ is adopted. In compact form this equation becomes the OrnsteinUhlenbeck equation

$$
d x=(\mathrm{A} x+\mathrm{B} u+\mathrm{C} v) d t+\mathrm{C} d \bar{w}
$$

where for notational convenience the superscripts $u, v$ are omitted from $x$. The well posedness of the state equation (3) follows from standard results in the theory of stochastic differential equations.

\subsection{The control objective}

We now define the control objective. Let us first fix a model, i.e. let us assume that the drift $v$ is fixed. Then, the control procedure is designed so that the distance form a desired target, chosen without loss of generality to be $x^{0}=0$ the zero sequence, is minimized at the minimum possible cost, as measured by the amplitude of the control variable $u$. Therefore, having chosen the state variable $x$ as given by the solution of the dynamic equation (3) the decision maker's goal is to choose the control procedure $u$ so as to solve the stochastic control problem ${ }^{6}$

$$
\min _{u} \mathbb{E}_{Q}\left[\int_{0}^{\infty} e^{-r t} \sum_{n, m}\left(p_{n m} x_{n}(t) x_{m}(t)+q_{n m} u_{n}(t) u_{m}(t)\right) d t\right]
$$

or in compact form

$$
\min _{u} \mathbb{E}_{Q}\left[\int_{0}^{\infty} e^{-r t}(\langle\mathrm{P} x(t), x(t)\rangle+\langle\mathrm{Q} u(t), u(t)\rangle) d t\right]
$$

where $\langle\cdot, \cdot\rangle$ is the inner product in the Hilbert space $\mathbb{R}^{N}$ and $\mathrm{P}, \mathrm{Q}: \mathbb{R}^{N} \rightarrow \mathbb{R}^{N}$ are symmetric positive operators, whose matrix representation is $\mathrm{P}=\left\{p_{n m}\right\}$ and $\mathrm{Q}=\left\{q_{n m}\right\}$ respectively.

\footnotetext{
${ }^{6}$ To simplify the exposition we do not consider, without loss of generality, cross product terms in the quadratic objective. Cross product terms can be eliminated by a change in units see for example Magill (1977b).
} 
In the special case of diagonal operators $p_{n m}=p \delta_{n m}, q_{n m}=q \delta_{n m}$ this functional assumes the simplified form

$$
\left.\min _{u} \mathbb{E}_{Q}\left[\int_{0}^{\infty} e^{-r t} \sum_{n} p\left(x_{n}(t)\right)^{2}+q\left(u_{n}(t)\right)^{2}\right) d t\right]
$$

The first sum, can be considered as the total deviation of the states of the system at each site from the desired state 0 whereas the second sum is the total control exerted on the system in the effort to drive it to 0 . We emphasize that because of the linearity of the system the choice of 0 as the target state is without any loss of generality whatsoever. This problem is solved under the adoption of the measure $Q$, related to the drift $v$, i.e. it is solved under the dynamic constraint (3). This will provide a solution leading to a value function $V\left(x_{0} ; v\right)$; corresponding to the minimum deviation obtained for the model $Q_{v}$ under the minimum possible effort. Being uncertain about the true model, the decision maker will opt to choose this strategy that will work in the worst case scenario; this being the one that maximizes $V\left(x_{0} ; v\right)$, the minimum over all $u$ having chosen $v$, over all possible choices for $v$. Therefore, the robust control problem to be solved is of the general form

$$
\min _{u} \max _{v} \mathbb{E}_{Q}\left[\int_{0}^{\infty} e^{-r t} \sum_{n} \sum_{m}\left(p_{n m} x_{n}(t) x_{m}(t)+q_{n m} u_{n}(t) u_{m}(t)-\theta r_{n m} v_{n}(t) v_{m}(t)\right) d t\right]
$$

or in compact form

$$
\min _{u} \max _{v} \mathbb{E}_{Q}\left[\int_{0}^{\infty} e^{-r t}(\langle(\mathrm{P} x)(t), x(t)\rangle+\langle(\mathrm{Q} u)(t), u(t)\rangle-\theta\langle(\mathrm{R} v)(t), v(t)\rangle) d t\right],
$$

subject to the dynamic constraint (3), where $\theta>0$ and $\mathrm{R}=\left\{r_{n m}\right\}$ is a symmetric positive operator. The third term corresponds to a quadratic loss function related to the "cost" of model misspecification. Quadratic loss functions are rather common in statistical decision theory, mainly on account of their connection with entropy (see Proposition 1).

In the special case where $p_{n m}=p \delta_{n m}, q_{n m}=q \delta_{n m}, r_{n m}=\delta_{n m}$ the cost functional simplifies to

$$
\left.\min _{u} \max _{v} \mathbb{E}_{Q}\left[\int_{0}^{\infty} e^{-r t} \sum_{n} p\left(x_{n}(t)\right)^{2}+q\left(u_{n}(t)\right)^{2}-\theta \sum_{n}\left(v_{n}(t)\right)^{2}\right) d t\right]
$$

subject to the dynamic constraint (3). The new term in the functional, is related to our aversion for model misspecification.

Another important special case is the case where $p_{n m}=p_{n} \delta_{n m}, q_{n m}=q_{n} \delta_{n m}, r_{n m}=\theta_{n} \delta_{n m}$ and $\theta=1$. Then the control functional simplifies to

$$
\left.\min _{u} \max _{v} \mathbb{E}_{Q}\left[\int_{0}^{\infty} e^{-r t} \sum_{n} p_{n}\left(x_{n}(t)\right)^{2}+q_{n}\left(u_{n}(t)\right)^{2}-\sum_{n} \theta_{n}\left(v_{n}(t)\right)^{2}\right) d t\right]
$$

subject to the dynamic constraint (3). This version of the control functional introduces localized concerns on the deviation from particular state targets, on the cost of required control as well as on the cost of model misspecification.

Remark 1 (Interpretation as a differential game). One particularly intuitive way of viewing this problem is as a two player game, the first player is the decision maker while the second player is nature who has control over the uncertainty. The first player chooses her actions so as to minimize the distance of the state of the system from a chosen target at the minimum possible cost, whereas the second player is a considered by the first player as a malevolent player who tries to mess up the first players efforts. This interpretation allows us to use the Hamilton-Jacobi-Bellman-Isaacs equation approach for the solution of the robust control problem. 


\subsection{Relation with entropic constrained robust control}

The optimization problem (5) subject to (3) for various choices of the operator R is related to entropic constrained robust control. We present here two examples where this assertion holds. The first example is related with a "global" in space entropy constraint, while the second example is related with a "localized" in space entropy constraint, which may be even more relevant in the robust control of spatially varying interconnected economic systems.

Proposition 1 (Global entropy constraints). The optimization problem (5) subject to (3), for the choice $R=I$, is related to a robust control problem with an entropic constraint of the form

$$
\begin{gathered}
\inf _{u} \sup _{Q \in \mathcal{Q}} \mathbb{E}_{Q}\left[\int_{0}^{\infty} e^{-r t}(\langle\mathrm{P} x(t), x(t)\rangle+\langle\mathrm{Q} u(t), u(t)\rangle) d t\right], \\
\text { subject to } \mathcal{H}(P \mid Q)<H_{0}
\end{gathered}
$$

and the dynamic constraint (3), where by $\mathcal{H}(P \mid Q)$ we denote the Kullback-Leibler entropy of the probability measures $P$ and $Q$.

Proof: Consider first a finite horizon problem with horizon $T>0$. Within the class of models considered, for any $T>0$, an application of the Girsanov theorem yields that the likelihood of the models is given by the Radon-Nikodym derivative of the measures $P$ and $Q$ in the form of equation (2) as long as the Novikov condition holds. A quick calculation yields that the relative entropy of $P$ and $Q$ is given by

$$
\mathcal{H}(Q \mid P):=\mathbb{E}_{Q}\left[\ln \left(\frac{d Q}{d P}\right)\right]=\frac{1}{2} \int_{0}^{T} \sum_{n} v_{n}^{2}(t) d t
$$

We now consider the robust optimization problem

$$
\begin{array}{ll}
\sup _{Q \in \mathcal{Q}} J(x, u ; v) & \\
\text { subject to } & \mathcal{H}(Q \mid P) \leq H_{0}
\end{array}
$$

and the dynamic constraint (3) where $J(x, u ; v):=\mathbb{E}_{Q}\left[\int_{0}^{T} e^{-r t}(\langle\mathrm{P} x(t), x(t)\rangle+\langle\mathrm{Q} u(t), u(t)\rangle) d t\right]$. The entropic constraint means that we are only considering models (i.e., measures $Q$ ) whose deviation in terms of the relative entropy from the "true" model (i.e., the measure $P$ ) is less than $H_{0}$. Taking into account the representation of the entropy in terms of $\left\{v_{n}\right\}$ and using Lagrange multipliers for the equivalent minimization problem $\inf _{Q \in \mathcal{Q}}(-J(x, u ; v))$ we see that a solution of the relative entropy constraint problem is equivalent to the solution of

$$
\inf _{Q \in \mathcal{Q}}-J(x, u ; v)+\theta\left(\mathcal{H}(Q \mid P)-H_{0}\right),
$$

subject to (3) where $\theta \in \mathbb{R}_{+}$plays the role of the Lagrange multiplier. This is of course equivalent to the maximization problem

$$
\sup _{Q \in \mathcal{Q}} J(x, u ; v)-\theta\left(\mathcal{H}(Q \mid P)-H_{0}\right)
$$

subject to (3) which using the representation of the relative entropy in terms of $\left\{v_{n}\right\}$ reduces to

$$
\sup _{\left\{v_{n}\right\}} \mathbb{E}_{Q}\left[\int_{0}^{T} e^{-r t}\left(\langle\mathrm{P} x(t), x(t)\rangle+\langle\mathrm{Q} u(t), u(t)\rangle-\theta \sum_{n} v_{n}^{2}(t)\right) d t\right],
$$


subject to (3). The above reasoning may explain the negative coefficient in front of the terms $\left\{v_{n}\right\}$. Taking the limit as $T \rightarrow \infty$ leads to the required result.

Generalizations of Proposition 1 for the case where more localized constraints with respect to model uncertainty are taken into account may be considered.

We motivate this by the following discussion: Assume that we are interested in the effect of uncertainty not on the Wiener process $w=\left\{w_{n}\right\}$ (the primary risk factors in our model) as such but rather on the process $W=\mathrm{T} w$ where $\mathrm{T}: \mathbb{R}^{N} \rightarrow \mathbb{R}^{N}$ is an appropriate operator. This means that we are not interested on the effect of model uncertainty on $\left\{w_{n}\right\}$ but on the linear combination $\left\{\sum_{m} t_{n m} w_{m}\right\}$, which is assumed to reflect more accurately the effect of noise on the state variable. Various choices for the operator $\mathrm{T}$ are possible. One obvious choice is $\mathrm{T}=\mathrm{C}$, this means that the policy maker is interested in specifying the uncertainty so that she may understand its effect on the state of the system at each lattice site. Another obvious choice is to take $\mathrm{T}$ defined as $\mathrm{T} w=w_{\ell}$ where $\ell \in \mathbb{Z}$; this choice means that the policy maker is not worried about the uncertainty with respect to the noise term in general, but only as far as the uncertainty at site $m$ is concerned.

Since $w$ is a Wiener process under $P$, it follows that $w_{n}(t) \sim N(0, t)$ while under $Q$ (as a consequence of Girsanov's theorem) $w_{n}(t) \sim N\left(-v_{n}, t\right)$. Therefore, by the properties of the normal distribution $W_{m} \sim N\left(0, \sum_{m} t_{n m} t_{n m}\right)$ under the measure $P$, whereas it is distributed as $W_{m} \sim$ $N\left(-\sum_{m} t_{n m} v_{m}, \sum_{m} t_{n m} t_{n m}\right)$ under the measure $Q$. Therefore, if we only consider the marginal measures $\bar{P}_{n}$ and $\bar{Q}_{n}$ which are related with the distributions of the random variable $W_{n}$ under the measures $P$ and $Q$ respectively, we may consider the "localized" entropy for the two measures as

$$
\bar{d}_{n}:=\mathcal{H}\left(\bar{Q}_{n}, \bar{P}_{n}\right)=\int_{0}^{T}\left(\sum_{m} t_{n m} v_{m}(t)\right)^{2} d t .
$$

The case where $\mathbf{T}$ is chosen such that $\mathrm{T} w=w_{n}$ (i.e. $\mathbf{T}=\pi_{n}$ the projection onto the lattice site $n$ ), then the local entropy is the entropy of the marginal measures $P_{n}, Q_{n}$ which give the distribution of the component $w_{n}$ given that $w$ is distributed with the measure $P$ and $Q$ respectively. In this case

$$
d_{n}:=\mathcal{H}\left(Q_{n}, P_{n}\right)=\int_{0}^{T} v_{n}^{2}(t) d t
$$

The robust control problem

$$
\inf _{u} \sup _{Q \in \mathcal{Q}} J(x ; u, v)
$$

subject to the localized entropy constraints $\bar{d}_{n} \leq H_{n}$ (where $\bar{d}_{n}$ is defined in (6)) or $d_{n} \leq H_{n}$ (where $d_{n}$ is defined in (7) may lead to optimal control problems of the form discussed here for proper choice of the operator R. As an illustration we provide the following proposition, which of course can be generalized to other choices for the constraints.

Proposition 2. The optimization problem (5) subject to (3), for the choice $\mathrm{R}=\mathrm{D}$, where $\mathrm{D}$ is a diagonal operator with representation $d_{n m}=\theta_{n} \delta_{n m}$ is related to a robust control problem with an entropic constraint of the form

$$
\begin{aligned}
& \inf _{u} \sup _{Q \in \mathcal{Q}} \mathbb{E}_{Q} {\left[\int_{0}^{\infty} e^{-r t}(\langle\mathrm{P} x(t), x(t)\rangle+\langle\mathrm{Q} u(t), u(t)\rangle) d t\right], } \\
& \text { subject to } \mathcal{H}\left(P_{n} \mid Q_{n}\right)<H_{n}, \quad n \in \mathbb{Z}
\end{aligned}
$$

and the dynamic constraint (3), where by $\mathcal{H}\left(P_{n} \mid Q_{n}\right)$ we denote the Kullback-Leibler entropy of the marginal probability measures $P_{n}$ and $Q_{n}$ (see equation 7)). 
Proof: The proof follows the same steps as that of Proposition 1 only that now we need the Lagrangian

$$
L=\sup _{v}\left\{J(x ; u, v)+\sum_{n} \theta_{n}\left(\mathcal{H}\left(\bar{Q}_{n} \mid \bar{P}_{n}\right)-H_{n}\right)\right\}
$$

where $\left\{\theta_{n}\right\}$ are the Lagrange multipliers needed in order to guarantee that the localized entropic constraints hold. The major difference with Proposition 1 is that here we need a sequence of Lagrange multipliers rather than a single Lagrange multiplier, since now the constraints are more than one. Using the expression (7) for the entropies, we obtain the stated results.

Remark 2. The introduction of the local entropic constraints means that the policy maker is concerned on the effect of model uncertainty on $W$ rather than on $w$, and her concerns differ at various lattice points. The concern of the policy maker on uncertainty at lattice site $n$ is quantified by $H_{n}$, the smaller $H_{n}$ is the less model uncertainty is she willing to accept for lattice site $n$, given her information about this site. This assumption is not unreasonable as certain lattice points may be considered as more crucial than others therefore specific care should be taken for them.

Remark 3. In the robust control problem of Proposition 2 the maximizing adversarial agent - Nature - chooses a $\left\{v_{n}(t)\right\}$ while $\theta_{n} \in\left(\underline{\theta}_{n},+\infty\right], \underline{\theta}_{n}>0$, is a penalty parameter restraining the maximizing choice of Nature. As noted above $\theta_{n}$ is associated with the Lagrange multiplier of the entropy constraint at each site. In the entropy constraint $H_{n}$ is the maximum misspecification error that the decision maker is willing to consider given the existing information about the system at site $n^{7}$. The lower bound $\underline{\theta}_{n}$ is a so-called breakdown point beyond which it is fruitless to seek more robustness because the adversarial (i.e. the maximizing) agent is sufficiently unconstrained so that she/he can push the criterion function to $+\infty$ despite the best response of the minimizing agent. Thus when $\theta_{n}<\underline{\theta}_{n}$ for a specific site robust control rules cannot be attained. In our terminology this site is a candidate for a "nucleus" of a hot spot since misspecification concerns for this site will break down robust control for the whole spatial domain. On the other hand when $\theta_{m} \rightarrow \infty$ or equivalently $H_{m}=0$ there are no misspecification concerns for this site and the benchmark model can be used. The effects of spatial connectivity can be seen in this extreme example. The spatial relation of site $m$ with site $n$ could break down regulation for both sites. If site $m$ was spatially isolated from $n$ there would have been no problem with regulation at $m$.

Remark 4. Alternative equivalent problems can be formulated. For instance one may consider utility maximization problems in lieu of distance from a target minimization problems. In such cases the agent wishes to maximize her utility while nature, the malevolent player, acts so as to minimize it. This corresponds to maximizing the worst case utility which formally leads to an equivalent problem with the max and the min interchanged. For uniformity and clarity of presentation we work throughout with the distance from a target minimization interpretation of the problem ( $\min / \max$ ) and emphasize that all our results may be easily modified to work for the utility maximization interpretations.

\section{Translation invariant systems: Closed form solution}

In this section we treat a special case of the robust control problem, which allows a solution in closed form. As discussed in Remark 7 the results in this section apply under rather restricted conditions ${ }^{8}$

\footnotetext{
${ }^{7}$ If the decision maker can use physical principles and statistical analysis to formulate bounds on the relative entropy of plausible probabilistic deviations from her/his benchmark model, these bounds can be used to calibrate the parameters $H_{n}$ (Athanassoglou and Xepapadeas (2012)).

${ }^{8}$ On the other hand are examples where the translation invariance property is relevant and useful, as in the longitudinal control and string stability of vehicular platoons, with $\mathfrak{L}=\mathbb{Z}$ (e.g. Bamieh et al. (2002) Curtain et al. (2008)). On more general terms translation invariance is quite commonly used in Interacting Particle Systems (IPS) type statistical mechanics models. Structures like IPS models are quite popular in modeling social interactions.
} 
however, the closed form solution allows us to obtain a good intuition concerning the qualitative behavior of the solution, which will guide us in the treatment of the general case in later sections.

Assume that the operators A, B and C are discrete convolution type operators. This is an assumption which essentially states that $a_{n m}=a_{n-m}$, i.e. the effect that a site $m$ has at site $n$ depends only on the distance between $n$ and $m$ and not on the actual positions of the sites. Therefore we assume that the operators $A, B$ and $C$ are translation invariant. This assumption allows us to make a great simplifying step towards the resolution of the problem. We employ the discrete Fourier transform on the lattice $\mathfrak{L}$, denoted by $\mathfrak{F}$ (for a detailed account of the Fourier transform the reader may consult Wong (2011)). The Fourier transform has the property of turning a convolution operator into a multiplication operator, i.e. $\mathfrak{F}(\mathrm{A} u)=\mathfrak{F}(\mathrm{A}) \mathfrak{F}(u)$ where by $\mathfrak{F}(\mathrm{A})$ we denote the Fourier transform of the matrix A. To ease notation we will use the convention $\hat{u}_{k}:=\mathfrak{F}(u)(k)$ where now $k$ takes values on the dual lattice. A similar notation with the hats will hold for all other involved quantities.

As the rationale for this section is simply to help us develop our intuition, and we plan to consider the problem in full generality in subsequent sections using techniques which are generally applicable, we will make a few more simplifying assumptions. We will assume that our physical space is the finite dimensional lattice $\mathbb{Z}_{N}$, so that the dynamical system is defined on the Hilbert space $\ell^{2}:=\ell^{2}\left(\mathbb{Z}_{N}\right)$ which is identified with $\mathbb{R}^{N}$ and furthermore we restrict our attention to the class of vectors in $\ell^{2}\left(\mathbb{Z}_{N}\right)$ such that their Fourier transforms are real valued vectors. This is done purely for simplicity and does not restrict the validity of our qualitative results. The case of the infinite lattice introduces several technicalities but also does not alter the qualitative nature of our results.

Definition 1. For $m=0, \cdots, N-1$ consider the following vectors which are elements of $\ell^{2}\left(\mathbb{Z}_{N}\right) \simeq \mathbb{R}^{N}$ :

$$
\mathfrak{C}^{(m)}:=\mathcal{R} e\left(\mathfrak{E}^{(m)}\right)=\left(1, \cos \left(2 \pi \frac{m}{N}\right), \cdots, \cos \left(2 \pi \frac{n m}{N}\right), \cdots, \cos \left(2 \pi \frac{(N-1) m}{N}\right)\right),
$$

and define

$$
\mathcal{X}_{R}:=\operatorname{span}\left(\mathfrak{C}^{(m)} ; m=0, \cdots, N-1\right) \subset \ell^{2}\left(\mathbb{Z}_{N}\right) \simeq \mathbb{R}^{N} .
$$

Remark 5. The space $\mathcal{X}_{R}$ contains vectors with specific symmetry patterns. For simplicity assume that $N=2 * n+1$ is odd. Since $\cos \left(2 \pi \frac{(N-r) m}{N}\right)=\cos \left(2 \pi \frac{r m}{N}\right)$ for all $r=1, \cdots, n$, any element $x$ of $\mathcal{X}_{R}$ is such that $x(0)$ is arbitrary whereas $x(1)=x(N-1), x(2)=x(N-2), \cdots, x(n)=x(n+1)=$ $x(2 n+1-n)$.

The following lemma is useful:

\section{Lemma 1.}

(i) If $x \in \mathcal{X}_{R}$ then $\operatorname{Im}(\hat{x})=0$.

(ii) Let $A$ be a symmetric matrix corresponding to a convolution operator (a circulant matrix) such that the first column of the matrix $A, a^{(1)} \in \mathcal{X}_{R}$. If $x \in \mathcal{X}_{R}$ then $\operatorname{Im}(\mathfrak{F}(A \star x))=0$.

Proof: The proof of (i) follows immediately from the linearity of the discrete Fourier transform and the properties of the vectors $\mathfrak{C}^{(m)}$. Then (ii) follows from the fact that $\mathfrak{F}(A \star x)=\mathfrak{F} a^{(1)} \mathfrak{F} x$, and each of the vectors involved in this product are real valued (by (i)).

We are now ready to state the assumptions needed for this section. We emphasize that these assumptions are only used here in order to provide a simple completely worked out example in order to motivate the general discussion that will be developed in subsequent sections of this paper.

\section{Assumption 1.}

(i) The operators A, B, C are translation invariant (such that they correspond to discrete convolution operators and are represented by circulant matrices). 
(ii) The first column of the matrix representation of these operators are vectors which belong to $\mathcal{X}_{R}$.

(iii) The initial condition $x_{0} \in \mathcal{X}_{R}$ and the stochastic process $w \in \mathcal{X}_{R}$.

Remark 6. Out of the above assumptions only (i) is essential for the treatment of the control problem using the Fourier transform. Assumptions (ii) and (iii) are adopted simply to make sure that the resulting dynamical system in Fourier space is real valued and thus facilitate the analysis. The results stated here, e.g., the treatment of the control problem using the Riccati equation by no means is restricted to the real valued case, and can be extended in the case where the resulting dynamical system is complex valued, by simple separation of the real and the imaginary parts. However, this would render the algebra rather involved, obscuring the main points regarding the qualitative behavior of the system, that we wish to stress here.

Example 1. There are many interesting operators arising in realistic models that satisfy Assumption 1 (ii). The discrete Laplacian is an example of such an operator. Furthermore copies of the identity operator are such operators as well. Therefore, an example that falls in this category is the case of system (3) with $\mathrm{A}=\Delta_{d}$, the discrete Laplacian operator defined (in 1 dimension) as $(\mathrm{A} x)_{n}=$ $x_{n+1}-2 x_{n}+x_{n-1}$ and $\mathrm{B}=b I, \mathrm{C}=c I$. This leads to diffusive effects on the lattice, but localized control and uncertainty effects. Other options are possible.

Proposition 3. Let $\mathrm{P}=p I, \mathrm{Q}=q I$, where $\mathbb{R}^{N} \rightarrow \mathbb{R}^{N}$ is the identity operator. Under Assumption 1 (i) the control system (5) under the dynamic constraint (3) decouples in Fourier space and becomes

$$
\left.\min _{\left\{\hat{u}_{k}\right\}} \max _{\left\{\hat{v}_{k}\right\}} \mathbb{E}_{Q}\left[\int_{0}^{\infty} e^{-r t} \sum_{k} p\left(\hat{x}_{k}(t)\right)^{2}+q\left(\hat{u}_{k}(t)\right)^{2}-\theta \sum_{n}\left(\hat{v}_{k}(t)\right)^{2}\right) d t\right]
$$

subject to the decoupled state equations

$$
d \hat{x}_{k}(t)=\left(\hat{a}_{k} \hat{x}_{k}(t)+\hat{b}_{k} \hat{u}_{k}(t)+\hat{c}_{k} \hat{v}_{k}(t)\right) d t+\hat{c}_{k} \sigma_{k} \mathfrak{w}_{k}(t), k \in \mathfrak{L},
$$

where $\hat{a}_{k}, \hat{b}_{k}, \hat{c}_{k}$ are the components of the Fourier transform of the first column of the matrix representation of the operators $\mathrm{A}, \mathrm{B}, \mathrm{C}$ respectively, and

$$
\sigma_{k}^{2}:=1+4 \sum_{r=1}^{n} \cos ^{2}\left(2 \pi \frac{r k}{N}\right)
$$

and $\mathfrak{w}_{k}$ is a standard Brownian motion under the measure $Q$.

If furthermore Assumptions 1 (ii) and (iii) hold then the robust control problem

$$
\left.\min _{\left\{\hat{u}_{k}\right\} \in \mathcal{X}_{R}} \max _{\left\{\hat{v}_{k}\right\} \in \mathcal{X}_{R}} \mathbb{E}_{Q}\left[\int_{0}^{\infty} e^{-r t} \sum_{k} p\left(\hat{x}_{k}(t)\right)^{2}+q\left(\hat{u}_{k}(t)\right)^{2}-\theta \sum_{n}\left(\hat{v}_{k}(t)\right)^{2}\right) d t\right]
$$

subject to the decoupled dynamic constraints admits real valued solutions in Fourier space.

Proof: Applying the Fourier transform $\mathfrak{F}$ on the equations for the state variables yields

$$
d \hat{x}_{k}(t)=\left(\hat{a}_{k} \hat{x}_{k}(t)+\hat{b}_{k} \hat{u}_{k}(t)+\hat{c}_{k} \hat{v}_{k}(t)\right) d t+\hat{c}_{k} \hat{w}_{k}(t), k \in \mathfrak{L} .
$$

Since $w \in \mathcal{X}_{R}$ it must possess the spatial symmetry of the elements of $\mathcal{X}_{R}$, therefore it consists of $n+1$ independent Wiener processes $w_{0}, w_{1}, \cdots, w_{n}$ and is of the form

$$
w(t)=\left(w_{0}, w_{1}, \cdots, w_{n}, w_{n}, w_{n-1}, \cdots, w_{1}\right)
$$


whose Fourier transform is real valued and equal to the vector $\hat{w}$ with coordinates

$$
\hat{w}_{k}(t)=w_{0}+2 \sum_{r=1}^{n} \cos \left(2 \pi \frac{r k}{N}\right)
$$

where by a simple application of Lévy's characterization theorem it can be seen that $\left\{\hat{w}_{k}\right\}=\sigma_{k} \mathfrak{w}$ where $\mathfrak{w}$ is a standard Wiener process with respect to the measure $Q$ and

$$
\sigma_{k}^{2}:=1+4 \sum_{r=1}^{n} \cos ^{2}\left(2 \pi \frac{r k}{N}\right)
$$

The system (9) is now a decoupled system and this greatly simplifies the presentation.

Assuming further that $\mathrm{P}=p I$ and $\mathrm{Q}=q I$ where $I: \mathbb{R}^{N} \rightarrow \mathbb{R}^{N}$ is the identity operator, so that we may use the Plancherel theorem to restate the control functional with respect to the Fourier transformed variables. According to this result,

$$
\sum_{n} u_{n}^{2}=\frac{1}{N} \sum_{k}[\mathfrak{F}(u)(k)]^{2}=\frac{1}{N} \sum_{k} \hat{u}_{k}^{2}
$$

where the first summation takes place in the lattice $\mathfrak{L}$ whereas the second summation takes place in the dual lattice. We have used the fact that we restrict our problem to control variables $u \in \mathcal{X}_{R}$ so that all the quantities involved in the Plancherel formula are real valued. In a similar fashion we may deal with the other quadratic terms.

Therefore, one may restate the control functional in Fourier space as

$$
\left.\mathcal{J}:=\frac{1}{N} \mathbb{E}_{Q}\left[\int_{0}^{\infty} e^{-r t} \sum_{k} p\left(\hat{x}_{k}(t)\right)^{2}+q\left(\hat{u}_{k}(t)\right)^{2}-\theta \sum_{k}\left(\hat{v}_{k}(t)\right)^{2}\right) d t\right]
$$

where using the Plancherel theorem we have replaced the summation over the primary lattice with the summation over the dual lattice. Notice that the effects of the size of the lattice (the $\frac{1}{N}$ terms) factor out and have a uniform effect over all the terms of the control functional.

The control problem then becomes

$$
\left.\min _{\left\{\hat{u}_{k}\right\}} \max _{\left\{\hat{v}_{k}\right\}} \mathbb{E}_{Q}\left[\int_{0}^{\infty} e^{-r t} \sum_{k} p\left(\hat{x}_{k}(t)\right)^{2}+q\left(\hat{u}_{k}(t)\right)^{2}-\theta \sum_{n}\left(\hat{v}_{k}(t)\right)^{2}\right) d t\right]
$$

subject to the decoupled state equations (9).

The decoupling of the system in Fourier space greatly facilitates its treatment and allows for explicit solutions.

Proposition 4. The solution of the robust control problem (8) subject to state constraints (9) is equivalent to the solution of the decoupled problems

$$
\left.\min _{\hat{u}_{k}} \max _{\hat{v}_{k}} \mathbb{E}_{Q}\left[\int_{0}^{\infty} e^{-r t} p\left(\hat{x}_{k}(t)\right)^{2}+q\left(\hat{u}_{k}(t)\right)^{2}-\theta \sum_{k}\left(\hat{v}_{k}(t)\right)^{2}\right) d t\right], \quad k \in \mathfrak{L}
$$

subject to the state constraint (9) for each individual $k \in \mathfrak{L}$.

Proof: The proof is straightforward and is omitted. 
Remark 7. The Fourier approach considered here is rather limited as to the class of problems it is applicable to. The two major limitations are (a) the assumption that the operators involved are translation invariant and (b) the use of the Plancherel formula (isometry) to turn the control functional from a mapping of the primal lattice to a mapping of the dual lattice. The second requirement limits considerably the type of control problems we are allowed to treat in this manner. As a result, only minor generalizations of the results of this section are allowed to systems of more general forms. For instance, under further restrictions on the operators, one could treat using Fourier transforms the localized entropic constrained problem introduced in Proposition 2 by defining the new variables $\bar{v}_{n}=\sqrt{\theta_{n}} v_{n}$ and rewriting the functional into a form where the Plancherel isometry holds. This is equivalent to transforming $v$ into $\bar{v}=\mathrm{D} v$, where $\mathrm{D}$ is a diagonal operator with representation $d_{n m}=\sqrt{\theta_{n}} \delta_{n m}$. However, this transformation changes the state equation as well, therefore care should be taken so that the operator $\mathrm{CD}^{-1}$ and $\mathrm{C}$ are at the same time translation invariant, so that the Fourier transform of the state equation is also diagonal. This remark shows the difficulties in generalizing the Fourier transform approach to systems of more general form. These difficulties are overcome in Section 5 where the general linear quadratic problem is treated via a different approach and not through the Fourier transform.

We will consider the solution of the above problems (both primal and dual) using dynamic programming techniques, through the use of the Hamilton-Jacobi-Bellman-Isaacs (HJBI) equation (see e.g. Isaacs (1999), Hansen and Sargent (2001), Anderson et al. (2003) and references therein).

Proposition 5 (Solution of primal problem). The solution of the primal problem

$$
\left.\min _{\hat{u}_{k}} \max _{\hat{v}_{k}} \mathbb{E}_{Q}\left[\int_{0}^{\infty} e^{-r t} p\left(\hat{x}_{k}(t)\right)^{2}+q\left(\hat{u}_{k}(t)\right)^{2}-\theta\left(\hat{v}_{k}(t)\right)^{2}\right) d t\right],
$$

subject to (9) for each $k$ is given by the optimal state equation

$$
d \hat{x}_{k}^{*}=R_{k} \hat{x}_{k}^{*} d t+\hat{c}_{k} \sigma_{k} d \mathfrak{w}_{k}
$$

where

$$
R_{k}:=\hat{a}_{k}-\frac{\hat{b}_{k}^{2} M_{2, k}}{2 q}+\frac{\hat{c}_{k}^{2} M_{2, k}}{2 \theta}
$$

and $M_{2, k}$ is the solution of

$$
\left(\frac{\hat{c}_{k}^{2}}{2 \theta}-\frac{\hat{b}_{k}^{2}}{2 q}\right) M_{2, k}^{2}+\left(2 \hat{a}_{k}-r\right) M_{2, k}+2 p=0 .
$$

The optimal controls are given by the feedback laws

$$
\hat{u}_{k}^{*}=-\frac{\hat{b}_{k} M_{2, k}}{2 q} \hat{x}_{k}^{*}, \quad \hat{v}_{k}^{*}=\frac{\hat{c}_{k} M_{2, k}}{2 \theta} \hat{x}_{k}^{*}
$$

Proof: Fix $k \in \mathbb{Z}$ and let $V_{k}$ be the value function corresponding to this choice. by

Let $\mathcal{L}_{k}: C^{2}(\mathbb{R}) \rightarrow C(\mathbb{R})$ be the generator operator of the diffusion process $\left\{\hat{x}_{k}(t)\right\}, t \in \mathbb{R}_{+}$defined

$$
\left(\mathcal{L}_{k} \Phi\right)\left(\hat{x}_{k}\right)=\left(\hat{a}_{k} \hat{x}_{k}+\hat{b}_{k} \hat{u}_{k}+\hat{c}_{k} \hat{v}_{k}\right) \frac{\partial \Phi}{\partial \hat{x}_{k}}+\frac{1}{2} \hat{c}_{k}^{2} \sigma_{k}^{2} \frac{\partial^{2} \Phi}{\partial \hat{x}_{k}^{2}} .
$$

The relevant Hamilton-Jacobi-Belman-Isaacs (HJBI) equation becomes

$$
r V_{k}=\bar{H}\left(\hat{x}_{k}, \frac{\partial V_{k}}{\partial \hat{x}_{k}}, \frac{\partial^{2} V_{k}}{\partial \hat{x}_{k}^{2}}\right)
$$


where for any function $\Phi$ of sufficient regularity the Hamiltonian $\bar{H}\left(\hat{x}_{k}, \frac{\partial \Phi}{\partial \hat{x}_{k}}, \frac{\partial^{2} \Phi}{\partial \hat{x}_{k}^{2}}\right)$ is defined by

$$
\bar{H}\left(\hat{x}_{k}, \frac{\partial \Phi}{\partial \hat{x}_{k}}, \frac{\partial^{2} \Phi}{\partial \hat{x}_{k}^{2}}\right):=\inf _{\hat{u}_{k}} \sup _{\hat{v}_{k}}\left(p \hat{x}_{k}^{2}+q \hat{u}_{k}^{2}-\theta \hat{v}_{k}^{2}+\mathcal{L}_{k} \Phi\right)
$$

and the optimization problems in the definition of the Hamiltonian are considered as static optimization problems over $\hat{v}_{k} \in \mathcal{V}_{k} \subset \mathbb{R}, \hat{u}_{k} \in \mathcal{U}_{k} \subset \mathbb{R}$, for fixed $k$, where $\mathcal{U}_{k}, \mathcal{V}_{k}$ are appropriate subsets of $\mathbb{R}$ 9 .

We first calculate $\bar{H}\left(x, \Phi_{x}, \Phi_{x x}\right)$ for any function $\Phi$, where we use the shorthand notation $\Phi_{x}=\frac{\partial \Phi}{\partial \hat{x}_{k}}$ and $\Phi_{x x}=\frac{\partial^{2} \Phi}{\partial \hat{x}_{k}^{2}}$ for simplicity.

The solution of the static optimization problem is given by the first order condition $\hat{v}_{k}^{*}=\frac{\hat{c}_{k}}{2 \theta} \Phi_{x}$. This corresponds to a maximum value which becomes

$$
\Psi:=\frac{\hat{c}_{k}^{2} \sigma_{k}^{2} \Phi_{x x}}{2}+q \hat{u}_{k}^{2}+p \hat{x}_{k}^{2}+b \Phi_{x} \hat{u}_{k}+\hat{a}_{k} \Phi_{x} \hat{x}_{k}+\frac{\hat{c}_{k}^{2} \Phi_{x}^{2}}{4 \theta} .
$$

We now minimize the function $\Psi$ with respect to $\hat{u}_{k}$. The first order condition for the minimum gives

$$
\hat{u}_{k}=-\frac{\hat{b}_{k} \Phi_{x}}{2 q}
$$

which upon substitution gives

$$
\bar{H}\left(x, \Phi_{x}, \Phi_{x x}\right)=\frac{\hat{c}_{k}^{2} \sigma_{k}^{2} \Phi_{x x}}{2}+p \hat{x}_{k}^{2}+\hat{a}_{k} \Phi_{x} \hat{x}_{k}-\frac{\hat{b}_{k}^{2} \Phi_{x}^{2}}{4 q}+\frac{\hat{c}_{k}^{2} \Phi_{x}^{2}}{4 \theta} .
$$

The HJBI equation thus assumes the form

$$
\frac{\hat{c}_{k}^{2} \sigma_{k}^{2} V_{x x}}{2}+p \hat{x}_{k}^{2}+\hat{a}_{k} V_{x} \hat{x}_{k}-\frac{\hat{b}_{k}^{2} V_{x}^{2}}{4 q}+\frac{\hat{c}_{k}^{2} V_{x}^{2}}{4 \theta}=r V
$$

which is a nonlinear second order differential equation.

We look for a solution of the special form

$$
V\left(\hat{x}_{k}\right)=\frac{M_{2, k}}{2} \hat{x}_{k}^{2}+M_{1, k} \hat{x}_{k}+M_{0, k}
$$

Substituting into the HJBI equation and matching coefficients of different orders of $\hat{x}_{k}$ we obtain that the coefficient $M_{i, k}, i=0,1,2$ are given by

$$
\begin{aligned}
& \left(\frac{\hat{c}_{k}^{2}}{2 \theta}-\frac{\hat{b}_{k}^{2}}{2 q}\right) M_{2, k}^{2}+\left(2 \hat{a}_{k}-r\right) M_{2, k}+2 p=0, \\
& M_{1, k}=0 \\
& M_{0, k}=\frac{\hat{c}_{k}^{2} \sigma_{k}^{2} M_{2, k}}{2 r} .
\end{aligned}
$$

The value function is thus obtained as long as the solution of the above quadratic equation is obtained.

We now substitute this expression into the equations for the optimal controls to obtain

$$
\hat{v}_{k}^{*}=\frac{\hat{c}_{k} M_{2, k}}{2|\theta|} \hat{x}_{k}, \quad \hat{u}_{k}^{*}=-\frac{\hat{b}_{k} M_{2, k}}{2 q} \hat{x}_{k} .
$$

\footnotetext{
${ }^{9}$ Or of $\mathbb{R}^{d}$ without loss of generality.
} 
Therefore, upon substitution into the state equation we see that the optimal state is given by the solution of the stochastic differential equation

$$
d \hat{x}_{k}^{*}=R_{k} \hat{x}_{k}^{*} d t+\hat{c}_{k} d \hat{w}_{k}
$$

where

$$
R_{k}:=\hat{a}_{k}-\frac{\hat{b}_{k}^{2} M_{2, k}}{2 q}+\frac{\hat{c}_{k}^{2} M_{2, k}}{2 \theta}
$$

This completes the proof.

Proposition 6 (Solution of the dual problem). The solution of problem

$$
\left.\max _{\hat{v}_{k}} \min _{\hat{u}_{k}} \mathbb{E}_{P}\left[\int_{0}^{\infty} e^{-r t} p\left(\hat{x}_{k}(t)\right)^{2}+q\left(\hat{u}_{k}(t)\right)^{2}-\theta\left(\hat{v}_{k}(t)\right)^{2}\right) d t\right], \quad k \in \mathfrak{L}
$$

subject to (9) for any $k \in \mathbb{Z}$ coincides with that of the primal problem (12) as given by Proposition 5 and there is no duality gap.

Proof: The value function of the dual problem $V^{\sharp}$ satisfies the Hamilton-Jacobi-Bellman equation

$$
\underline{H}\left(\hat{x}_{k}, V_{x}^{\sharp}, V_{x x}^{\sharp}\right)=r V^{\sharp}
$$

where for any function $\Phi$, of sufficient regularity, the Hamiltonian $\underline{H}\left(x, \Phi_{x}, \Phi_{x x}\right)$ is defined by

$$
\underline{H}\left(x, \Phi_{x}, \Phi_{x x}\right):=\sup _{\hat{v}_{k}} \inf _{\hat{u}_{k}}\left\{p \hat{x}_{k}^{2}+q \hat{u}_{k}^{2}-\theta \hat{v}_{k}^{2}+\mathcal{L}_{k} \Phi\right\}
$$

where $\mathcal{L}_{k}$ is the generator operator of the diffusion process, defined by (14). As before the optimization problem is a static one. A quick calculation shows that for any function $\Phi$,

$$
\underline{H}\left(x, \Phi_{x}, \Phi_{x x}\right)=\bar{H}\left(x, \Phi_{x}, \Phi_{x x}\right)
$$

thus leading to the same HJBI equation as for the primal problem. The result then follows retracing the steps in the proof of Proposition 5.

Remark 8. The above two propositions simply provide candidates for the solution of the problem. Whether these candidates are indeed solutions and whether the solution is a saddle point depends on the choice of the parameter $\theta$, as will become clear in the next section.

Remark 9 (Certainty equivalent). Suppose that instead of the stochastic problem treated here we treat instead the control problem with the deterministic state equation

$$
d \hat{x}_{k}=\left(\hat{a}_{k} \hat{x}_{k}+\hat{b}_{k} \hat{u}_{k}+\hat{c}_{k} \hat{v}_{k}\right) d t
$$

and the same quadratic cost functional (where of course now the expectation is redundant). This is a deterministic linear quadratic optimal control system. The solution of the relevant robust control problem is governed by the Hamilton-Jacobi-Isaacs equation, where the operator $\mathcal{L}_{k}$ is now replaced by the first order operator $\mathcal{L}_{k}^{0}$ with the following action on the value function: $\mathcal{L}_{k}^{0} V=\left(\hat{a}_{k} \hat{x}_{k}+\hat{b}_{k} \hat{u}_{k}+\right.$ $\left.\hat{c}_{k} \hat{v}_{k}\right) V_{x}$. Then, working in the same fashion as in Propositions 5 and 6 we see that the optimal policy for the deterministic problem coincides with that of the stochastic problem. Therefore, as far as the form of the feedback law of the optimal policies are concerned stochastic effects play no role. This has been called by Hansen and Sargent the certainty equivalent. Our results suggest that this certainty equivalence result can be extended to translation invariant spatial systems. However, one should be extremely cautious with that, since in the stochastic case the optimal policy is a stochastic process (through the dependence of $u^{*}$ and $v^{*}$ on $x$ which is a stochastic process. On the contrary, the optimal policy in the deterministic case is deterministic (through the dependence of $u^{*}$ and $v^{*}$ on $x$ which is a deterministic process). This qualitative behavior shows in the calculation of the value function, which for the certainty equivalent problem works out to be $V^{0}=\frac{M_{2, k}}{2} \hat{x}_{k}^{2}<V$. 


\section{Hot spot formation in translation invariant systems}

In this section we study the validity and the qualitative behavior of the controlled system. We will call the qualitative changes of the behavior of the system hot spots. We will define three types of hot spots:

$\triangleright$ Hot spot of type I: This is a breakdown of the solution procedure, i.e., a set of parameters where a solution to the above problem does not exist.

$\triangleright$ Hot spot of type II: This corresponds to the case where the solution exists but may lead to spatial pattern formation, i.e., to spatial instability similar to the Turing instability.

$\triangleright$ Hot spot of type III: This corresponds to the case where the cost of robustness becomes more that what is offering us, i.e., where the relative cost of robustness may become very large.

In what follows we discuss the formation of hot spots in the case of finite lattices $\mathbb{Z}_{N}$; the mechanism for hot spot formation in the infinite lattice is similar and certain remarks will be made when necessary.

\subsection{Hot spots of type I}

The breakdown of the solution procedure can be seen quite easily by the following simple argument. As seen in the proof of Proposition 5 the value function assumes a simple quadratic form, as long as the algebraic quadratic equation

$$
\left(\frac{\hat{c}_{k}^{2}}{2 \theta}-\frac{\hat{b}_{k}^{2}}{2 q}\right) M_{2, k}^{2}+\left(2 \hat{a}_{k}-r\right) M_{2, k}+2 p=0 .
$$

admits real valued solutions, at least one of which is positive. The positivity of the real root is needed since, by general considerations in optimal control, the value function must be convex. If the above algebraic quadratic equation does not admit at least one positive real valued solution this is an indication of breakdown of the existence of a solution to the robust control problem which will be called a hot spot of Type I.

Proposition 7 (Type I hot spot creation:). Hot spots of Type I may be created in one of the following two cases:

$\left(I_{A}\right)$ Either,

$$
\left(2 \hat{a}_{k}-r\right)^{2}<8 p\left(\frac{\hat{c}_{k}^{2}}{2 \theta}-\frac{\hat{b}_{k}^{2}}{2 q}\right)
$$

$\left(I_{B}\right)$ Or,

$$
\left(2 \hat{a}_{k}-r\right)^{2}>8 p\left(\frac{\hat{c}_{k}^{2}}{2 \theta}-\frac{\hat{b}_{k}^{2}}{2 q}\right),\left(\frac{\hat{c}_{k}^{2}}{2 \theta}-\frac{\hat{b}_{k}^{2}}{2 q}\right)>0,2 \hat{a}_{k}-r>0 .
$$

Remark 10. Hot spots of this type may arise either due to low values of $\theta$, or due to high values of $q$ or low values of $r$. For example, they may arise either if

$$
\theta<\frac{p \hat{c}_{k}^{2}}{\left(\hat{a}_{k}-\frac{r}{2}\right)^{2}+\frac{p}{q} \hat{b}_{k}^{2}}, \quad k \in \mathbb{Z}_{N}
$$


or if

$$
\theta>\frac{p \hat{c}_{k}^{2}}{\left(\hat{a}_{k}-\frac{r}{2}\right)^{2}+\frac{p}{q} \hat{b}_{k}^{2}}, \quad \frac{q}{\theta}>\frac{\hat{b}_{k}^{2}}{\hat{c}_{k}^{2}}, \quad r<2 \hat{a}_{k}, \quad k \in \mathbb{Z}_{N} .
$$

In particular hot spots are expected to occur in the limit as $\theta \rightarrow 0$ while they are not expected to occur in the limit as $\theta \rightarrow \infty$.

Proof of Proposition 7: Let us rewrite the above equation in the simpler form

$$
M_{2, k}^{2}+\frac{\mathfrak{a}}{\mathfrak{R}}+\frac{2 p}{\mathfrak{R}}=0
$$

where

$$
\mathfrak{a}:=2 \hat{a}_{k}-r, \quad \mathfrak{R}:=\frac{\hat{c}_{k}^{2}}{2 \theta}-\frac{\hat{b}_{k}^{2}}{2 q},
$$

assuming of course that $\mathfrak{R} \neq 0$. The condition for having two real solutions of equation (16) is that the discriminant is positive,

$$
\Delta:=\left(\frac{\mathfrak{a}}{\mathfrak{R}}\right)^{2}-8 \frac{p}{\mathfrak{R}}>0
$$

which is equivalent to the condition $\mathfrak{a}^{2}>8 p \mathfrak{R}$. If $\mathfrak{R}<0$ this condition always holds, whereas if $\mathfrak{R}>0$ it will hold for particular modes which of course depend on the parameter values. We now look for the solutions. We will consider two cases and the relevant subcases:

- Case A: $\mathfrak{R}<0$. Then $\sqrt{\Delta} \geq|\mathfrak{a} / \mathfrak{R}|$.

- Case A1: If $\mathfrak{a}>0$ then there is only one positive solution which is

$$
M_{2, k}=\frac{1}{2}\left(-\frac{\mathfrak{a}}{\mathfrak{R}}+\sqrt{\Delta}\right)
$$

- Case A2: If $\mathfrak{a}<0$ then there is only one positive solution (which is exactly of the same form as in Case A1),

$$
M_{2, k}=\frac{1}{2}\left(-\frac{\mathfrak{a}}{\mathfrak{R}}+\sqrt{\Delta}\right)
$$

- Case B: $\mathfrak{R}>0$. Then $\sqrt{\Delta} \leq|\mathfrak{a} / \mathfrak{R}|$.

- Case B1: If $\mathfrak{a}>0$ then there is two negative solutions none of which is acceptable on account of loss of convexity of the value function, therefore this is a hot spot of Type I.

- Case B2: If $\mathfrak{a}<0$, then there are two positive solutions

$$
0 \leq M_{2, k}^{(1)}=\frac{1}{2}\left(-\frac{\mathfrak{a}}{\mathfrak{R}}-\sqrt{\Delta}\right) \leq M_{2, k}^{(2)}=\frac{1}{2}\left(-\frac{\mathfrak{a}}{\mathfrak{R}}+\sqrt{\Delta}\right) .
$$

Out of these two we should keep the smaller one $M_{2, k}^{(1)}$ which of course will give the minimum value function (the other choice will correspond to a "second best" or suboptimal solution).

Therefore, summarizing a hot spot of Type I may arise if

$\left(I_{A}\right)$ Either, $\mathfrak{a}^{2}<8 p \mathfrak{R}$

$\left(I_{B}\right)$ Or, $\mathfrak{a}^{2}>8 p \mathfrak{R}, \mathfrak{R}>0$ and $\mathfrak{a}>0$.

or in terms of the original notation if 
$\left(I_{A}\right)$ Either,

$$
\left(2 \hat{a}_{k}-r\right)^{2}<8 p\left(\frac{\hat{c}_{k}^{2}}{2 \theta}-\frac{\hat{b}_{k}^{2}}{2 q}\right)
$$

$\left(I_{B}\right)$ Or,

$$
\left(2 \hat{a}_{k}-r\right)^{2}>8 p\left(\frac{\hat{c}_{k}^{2}}{2 \theta}-\frac{\hat{b}_{k}^{2}}{2 q}\right),\left(\frac{\hat{c}_{k}^{2}}{2 \theta}-\frac{\hat{b}_{k}^{2}}{2 q}\right)>0,2 \hat{a}_{k}-r>0 .
$$

Conditions (20) and (21) for the occurrence of hot spots of Type I are multiparameter conditions which are easily checked once a particular system is selected for study but when trying to infer general qualitative aspects concerning the optimal path there is not a simple or unique way of interpreting them. They may hold for some $k \in \mathbb{Z}$, meaning that the robust control procedure will break down in the particular site of the dual lattice, thus bringing down the successful control procedure of the whole system due to the coupling effects. One may call that mechanism a transmission of breakdown. Another way to look at these conditions is to use them as selection criteria for the parameters of the system not related to the operators $A, B, C$ for which a hot spot will definitely occur. For example case $I_{A}$ can be translated to

$$
\theta<\frac{p \hat{c}_{k}^{2}}{\left(\hat{a}_{k}-\frac{r}{2}\right)^{2}+\frac{p}{q} \hat{b}_{k}^{2}}, \quad k \in \mathbb{Z}_{N}
$$

which will hold for every site in the dual lattice as long as

$$
\theta<\theta_{c r}:=\min _{k \in \mathbb{Z}_{N}}\left\{\frac{p \hat{c}_{k}^{2}}{\left(\hat{a}_{k}-\frac{r}{2}\right)^{2}+\frac{p}{q} \hat{b}_{k}^{2}}\right\} .
$$

Therefore, if $\theta$ is too small, smaller than the critical value $\theta_{c r}$ then the robust control mechanism breaks down and Type I hot spots will certainly occur. However, this is not the only possible case. As Case $I_{B}$ shows, if

$$
\theta>\frac{p \hat{c}_{k}^{2}}{\left(\hat{a}_{k}-\frac{r}{2}\right)^{2}+\frac{p}{q} \hat{b}_{k}^{2}}, \quad q>\theta \frac{\hat{b}_{k}^{2}}{\hat{c}_{k}^{2}}, \quad r<2 \hat{a}_{k}, \quad k \in \mathbb{Z}_{N},
$$

then a hot spot of Type I will arise. This will be true if

$$
\theta>\max _{k \in \mathbb{Z}_{N}}\left\{\frac{p \hat{c}_{k}^{2}}{\left(\hat{a}_{k}-\frac{r}{2}\right)^{2}+\frac{p}{q} \hat{b}_{k}^{2}}\right\}, \quad q>\max _{k \in \mathbb{Z}_{N}}\left\{\theta \frac{\hat{b}_{k}^{2}}{\hat{c}_{k}^{2}}\right\}, \quad r<2 \min _{k \in \mathbb{Z}_{N}}\left\{\hat{a}_{k}\right\},
$$

meaning that hot spots of Type I may also arise for high values of $\theta$ if either the cost of control is high or if the discount factor is low enough.

Remark 11 (Hot spot of type I and loss of convexity). As mentioned above, a hot spot of Type I represents breakdown of the solvability of the optimal control problem. We argue that this represents some sort of loss of convexity of the problem thus leading to non existence of solution. To illustrate this point more clearly let us take the limit as $\theta \rightarrow 0$ which corresponds to hot spot formation. For such values of $\theta$, the particular ansatz employed for the solution breaks down and in fact as $\theta \rightarrow 0$ we expect $M_{2, k} \rightarrow 0$ so that the quadratic term in the value function will disappear. This leads to loss of strict concavity of the functional, which may be seen as follows: The functional contains a contribution from $\hat{v}_{k}$ through the dependence of $\hat{x}_{k}$ on $\hat{v}_{k}$ which contributes a quadratic term of positive sign in $\hat{v}_{k}$. The robustness term, which is proportional to $-\theta$ contributes a quadratic term of negative sign 
in $\hat{v}_{k}$. For large enough values of $\theta$ the latter term dominates in the functional and guarantees the strict concavity, therefore, leading to a well defined maximization problem. In the limit of small $\theta$ the former term dominates and thus turn the functional into a convex functional leading to problems with respect to the maximization problem over $\left\{\hat{v}_{k}\right\}$. We call this breakdown of concavity in $v$, which lead to loss of convexity of the value function in $x$, for small values of $\theta$ a hot spot of type I. When this happens, there is a duality gap, since the assumptions of the min-max theorem do not hold. In terms or regulatory objectives this means that concerns about model misspecification make regulation impossible.

The following examples show some interesting limiting situations:

Example 2. Assume that $A$ is the discrete Laplacian whereas $B$ and $C$ are copies of the identity operator. This corresponds to the case that there is diffusive coupling in the state equation but controls as well as the uncertainty have purely localized effects. A quick calculation shows that in this case $a_{k}=\alpha\left(1+2 \cos \left(\frac{2 \pi k}{N}\right)\right)$ where $\alpha$ is the diffusion coefficient whereas $b_{k}=\beta$ and $c_{k}=\gamma$ for every $k \in \mathbb{Z}_{N}$ where $\beta$ and $\gamma$ is a measure for the control and the uncertainty respectively. In this particular case, the quadratic equation becomes

$$
\left(\frac{\gamma^{2}}{2 \theta}-\frac{\beta^{2}}{2 q}\right) M_{2, k}^{2}+\left(2 \alpha\left(1+2 \cos \left(\frac{2 \pi k}{N}\right)\right)-r\right) M_{2, k}+2 p=0 .
$$

which must have a real valued solution for every $k$. There will not exist real valued solutions if

$$
\Delta:=\left(2 \alpha\left(1+2 \cos \left(\frac{2 \pi k}{N}\right)\right)-r\right)^{2}-8 p\left(\frac{\gamma^{2}}{2 \theta}-\frac{\beta^{2}}{2 q}\right)<0
$$

or equivalently after some algebra

$$
\left(\left(1+2 \cos \left(\frac{2 \pi k}{N}\right)\right)^{2}-\frac{r}{2 \alpha}\right)<\frac{p}{\alpha^{2}}\left(\frac{\gamma^{2}}{\theta}-\frac{\beta^{2}}{q}\right) .
$$

This is the condition for generation of a hot spot of Type I in this particular example. If this condition holds for some $k \in \mathbb{Z}_{N}$, this particular $k$ is a candidate for such a hot spot. We may spot directly that this cannot hold for any $k \in \mathbb{Z}_{N}$ if the right hand side of this inequality is negative, i.e., when $\theta>\theta_{c r}:=q \frac{\gamma^{2}}{\beta^{2}}$, therefore hot spots of this type will never occur for large enough values of $\theta$. The critical value of $\theta$ for the formation of such hot spots will depend on the relative magnitude of uncertainty over control . For $\theta<\theta_{c r}$ then a hot spot of Type I may occur for the modes $k$ such that

$$
\left(1+2 \cos \left(\frac{2 \pi k}{N}\right)\right)^{2} \leq \frac{r}{2 \alpha}+\rho
$$

or equivalently for $k$ such that

$$
\left(1+2 \cos \left(\frac{2 \pi k}{N}\right)\right)^{2} \leq\left(\frac{r}{2 \alpha}+\rho\right)^{\frac{1}{2}}
$$

where $\rho^{2}=\frac{p}{\alpha^{2}}\left(\frac{\gamma^{2}}{\theta}-\frac{\beta^{2}}{q}\right)$.

Example 3. The opposite case is when $A$ is again the discrete Laplacian while $B$ and $C$ are multiples of matrices containing 1 in the diagonal and the same entry $\nu$ in every other position. This means that the controls as well as the uncertainty has a globalized effect to all lattice points, in the sense that the controls even at remote lattice sites have an effect at each lattice point. Then $\hat{b}_{k}=\beta \delta_{k, 0}$, 
$\hat{c}_{k}=\gamma \delta_{k, 0}$, i.e., the Fourier transform is fully localized and is a delta function. Then, for $k=0$ the quadratic equation becomes

$$
\left(\frac{\gamma^{2}}{2 \theta}-\frac{\beta^{2}}{2 q}\right) M_{2,0}^{2}-(6 \alpha-r) M_{2,0}+2 p=0
$$

while for $k \neq 0$ the quadratic term vanishes yielding

$$
-\left(2 \alpha\left(1+2 \cos \left(\frac{2 \pi k}{N}\right)\right)-r\right) M_{2,0}+2 p=0
$$

\subsection{Hot spots of type II}

We now consider the spatial behavior of the optimal path, as given by the Itō stochastic differential equation

$$
d \hat{x}_{k}^{*}=R_{k} \hat{x}_{k}^{*} d t+\hat{c}_{k} d \hat{w}_{k}
$$

The optimal path is a random field, thus leading to random patterns in space, some of which may be short lived and generated simply by the fluctuations of the Wiener process. We thus look for the spatial behavior of the mean field as describable by the expectation $\hat{X}_{k}:=\mathbb{E}_{Q}\left[\hat{x}_{k}^{*}\right]$. By standard linear theory $\hat{X}_{k}(t)=\hat{X}_{k}(0) \exp \left(R_{k} t\right)$ and this means that for the modes $k \in \mathbb{Z}_{N}$ such that $R_{k} \geq 0$ we have temporal growth and these modes will dominate the long term temporal behavior. On the contrary modes $k$ such that $R_{k}<0$ decay as $t \rightarrow \infty$ therefore such modes correspond to (short term) transient temporal behavior, not likely to be observable in the long term temporal behavior. The above discussion implies that the long time asymptotic of the solution in Fourier space will be given by

$$
\hat{X}_{k}(t) \simeq\left\{\begin{array}{cc}
\hat{x}_{k}(0) \exp \left(R_{k} t\right), & k \in \mathcal{P}:=\left\{k \in \mathbb{Z}_{N}: R_{k} \geq 0\right\} \\
0 & \text { otherwise }
\end{array}\right.
$$

To see what this pattern will look like in real space, we simply need to invert the Fourier transform, thus obtaining a spatial pattern of the form

$$
X_{n}(t):=\mathbb{E}_{Q}\left[x_{n}(t)\right]=\sum_{k \in \mathcal{P}} \hat{x}_{k}(0) \exp \left(R_{k} t\right) \cos \left(2 \pi \frac{k}{N} n\right) .
$$

The above discussion therefore leads us to a very important conclusion, which is of importance to economic theory of spatially interconnected systems:

If as an effect of the robust optimal control procedure exerted on the system there exist modes $k \in \mathbb{Z}_{N}$ such that $R_{k}>0$, then this will lead to spatial pattern formation which will create spatial patterns of the form (22). As we will see there are cases what such patterns will not exist in the uncontrolled system and will appear as an effect of the control procedure. We will call such patterns an optimal robustness induced spatial instability or hot spot of Type II.

The economic significance of this result should be stressed. We show the emergence of a spatial pattern formation instability, which can be triggered by the optimal control procedures exerted on the system; in other words emergence of spatial clustering and agglomerations in the economy caused by uncertainty aversion and robust control. This observation can further be extended in the case of nonlinear dynamics, in the weakly nonlinear case. When the dynamics are nonlinear in the state the emergence of hot spots of Type II and optimal robustness induced spatial instability should be linked to the spatial instability of a spatially uniform steady state corresponding to the linear quadratic approximation of a nonlinear system. This instability which can be thought as pattern formation 
precursor will induce the emergence of spatial clustering. As time progresses and the linearized solution (22) grows beyond a certain critical value (in terms of a relevant norm) then the deviation from the homogeneous steady state is so large that the linearized dynamics are no longer a valid approximation. Then the nonlinear dynamics will take over and as an effect of that some of the exponentially growing modes could be balanced thus leading to more complicated stable patterns. At any rate even in the nonlinear case the mechanism described here will be a Turing type pattern formation mechanism explaining the onset of spatial patterns in the economy ${ }^{10}$.

The next proposition identifies which modes can lead to hot spot of Type II formation (optimal robustness induced spatial instability) and in this way through equation (22) identifies possible spatial patterns that can emerge in the spatial economy.

Proposition 8 (Pattern formation for the primal problem). There exist pattern formation behavior for the primal problem if there exist modes $k$ such that $R_{k}>0$, i.e., if there exist modes $k$ such that

$$
\begin{aligned}
& \frac{1}{2}\left(r-\sqrt{r^{2}+8 p\left(\frac{\hat{c}_{k}^{2}}{2 \theta}-\frac{\hat{b}_{k}^{2}}{2 q}\right)}\right) \leq \hat{a}_{k} \leq \frac{1}{2}\left(r+\sqrt{r^{2}+8 p\left(\frac{\hat{c}_{k}^{2}}{2 \theta}-\frac{\hat{b}_{k}^{2}}{2 q}\right)}\right), \\
& r^{2}+8 p\left(\frac{\hat{c}_{k}^{2}}{2 \theta}-\frac{\hat{b}_{k}^{2}}{2 q}\right) \geq 0 .
\end{aligned}
$$

Proof: The expectation $X_{k}^{*}:=\mathbb{E}_{Q}\left[x_{k}^{*}\right], k \in \mathbb{Z}$ of the optimal path is given by the solution of the linear deterministic ordinary differential equation $d X_{k}^{*}(t)=R_{k} X_{k}^{*}(t) d t, k \in \mathbb{Z}$. Thus pattern formation occurs for these $k \in \mathbb{Z}$ such that $R_{k}>0$. Let us now try to express $R_{k}$ in a form which reveals in a more clear fashion the actual dynamics of the optimal path. We will use the notation of Section 4.1, introducing again the quantities $\mathfrak{a}$ and $\mathfrak{R}$ (see equation (19)). In terms of these quantities we rewrite $R_{k}=\hat{a}_{k}+\mathfrak{R} M_{2, k}$ where $M_{2, k}$ is the positive solution of the quadratic equation (of course we assume that we do not have occurrence of a Type I hot spot). We are limited in cases $A 1, A 2$ and $B 2$ of Section 4.1. A quick calculation shows that in cases $A 1$ and $A 2$,

$$
R_{k}=\frac{1}{2}(r+\mathfrak{R} \sqrt{\Delta})
$$

whereas in case $B 2$,

$$
R_{k}=\frac{1}{2}(r-\mathfrak{R} \sqrt{\Delta})
$$

where

$$
\Delta:=\left(\frac{\mathfrak{a}}{\mathfrak{R}}\right)^{2}-8 \frac{p}{\mathfrak{R}}>0 .
$$

Note that in any case $R_{k}<\frac{r}{2}$ which is of course expected since on the optimal path the functional is finite therefore possible exponential growth of a mode cannot exceed $e^{\frac{r}{2} t}$. Thus pattern formation type behavior in the optimal path will correspond to cases where $0 \leq R_{k}<\frac{r}{2}$. Since the right hand side of the inequality always holds, we just consider the left hand side. A simple but tedious algebraic calculation (nor reproduced here) shows that in any of the above cases $R_{k} \geq 0$ implies $\mathfrak{a}^{2} \leq r^{2}+8 p \Re$ (which can only hold as long as $r^{2}+8 \pi \mathfrak{R} \geq 0$ ) and this is equivalent to

$$
\frac{1}{2}\left(r-\sqrt{r^{2}+8 p \Re}\right) \leq \hat{a}_{k} \leq \frac{1}{2}\left(r-+\sqrt{r^{2}+8 p \Re}\right) .
$$

\footnotetext{
${ }^{10}$ For more details concerning the connection (similarities and differences) of the optimal robustness induced instability with the Turing mechanism for pattern formation see Remark 16. For a full discussion of the nonlinear case (beyond the particular case of translation invariant operators) see Sections 7 and 6 .
} 
Thus modes satisfying this condition will lead to pattern formation. This condition translated to the original parameters of the problem yields the pattern formation condition

$$
\begin{aligned}
& \frac{1}{2}\left(r-\sqrt{r^{2}+8 p\left(\frac{\hat{c}_{k}^{2}}{2 \theta}-\frac{\hat{b}_{k}^{2}}{2 q}\right)}\right) \leq \hat{a}_{k} \leq \frac{1}{2}\left(r+\sqrt{r^{2}+8 p\left(\frac{\hat{c}_{k}^{2}}{2 \theta}-\frac{\hat{b}_{k}^{2}}{2 q}\right)}\right), \\
& r^{2}+8 p\left(\frac{\hat{c}_{k}^{2}}{2 \theta}-\frac{\hat{b}_{k}^{2}}{2 q}\right) \geq 0 .
\end{aligned}
$$

It is interesting to see what is the behavior of the system as a function of parameters with respect to pattern formation and the qualitative behavior of the optimal path.

Remark 12 (Pattern formation and the discount factor). Note that this pattern formation behavior is in full accordance with the fact that our state equation is the optimal path for the linear quadratic control problem. Since it solves this problem it is guaranteed that $I:=\mathbb{E}_{Q}\left[\int_{0}^{\infty} e^{-r t} \hat{x}_{k}^{2}(t) d t\right]$ is finite ${ }^{11}$ therefore $\hat{x}_{k}(t)$ can at most grow as $e^{\frac{r}{2} t}$, otherwise the quantity $I$ would be infinite. This is verified explicitly in the proof of the above proposition where it is shown that $R_{k} \leq \frac{r}{2}$ for every $k \in \mathbb{Z}_{N}$. Therefore, all possible patterns may at most exhibit growth rates less or equal to $r / 2$. In the limit as $r \rightarrow 0$ i.e. in the limit of small discount rates pattern formation is becoming increasingly difficult in the linear quadratic model since growing patterns will be suppressed by the control procedures.

Proposition 9 (Stabilizing or destabilizing effects of control). The robust control procedure may either have a stabilizing or destabilizing effect with respect to pattern formation. in the sense that it may either stabilize an unstable mode of the uncontrolled system or on the contrary facilitate the onset of instabilities.

In particular,

(i) If $\frac{q}{\theta}<\frac{\hat{b}_{k}^{2}}{\hat{c}_{k}^{2}}$ then the robust control procedure has a stabilizing effect

(ii) If $\frac{q}{\theta}>\frac{\hat{b}_{k}^{2}}{\hat{c}_{k}^{2}}$ then the robust control procedure has a destabilizing effect

Case (ii) suggests robust control caused pattern formation, in the sense that we obtain a growing mode leading to a pattern which would not have appeared in the uncontrolled system.

Proof: To support the above claim we need to compare the threshold in $\hat{a}_{k}$ for the onset of instability in the uncontrolled system $a_{k, c r}^{(0)}$ and the relevant quantity $a_{k, c r}^{(c)}$. Of course for the uncontrolled system $a_{k, c r}^{(0)}$, whereas by Proposition 8 we see that $a_{k . c r}^{(0)}:=\frac{1}{2}\left(r-\sqrt{r^{2}+8 p\left(\frac{\hat{c}_{k}^{2}}{2 \theta}-\frac{\hat{b}_{k}^{2}}{2 q}\right)}\right)$. If $a_{k, c r}^{(c)}>a_{k, c r}^{(0)}=0$ then control has a stabilizing effect over mode $k$ (since the effect of control is to make it more difficult for this mode to develop instability by raising the instability threshold) while if on the contrary $a_{k, c r}^{(c)}<a_{k, c r}^{(0)}=0$, then control has a de-stabilizing effect over mode $k$ (since the effect of control is to make it easier for this mode to develop instability by lowering the instability threshold). Then by simple algebra the claim arises.

Remark 13. However, it should be emphasized that for any parameter values the robust control imposes an upper bound of $\frac{r}{2}$ for any unstable mode (see also Remark 12) whereas this does not hold for the uncontrolled system, for which unstable modes may have any growth rate, determined purely by the spectrum of $A$ (the largest growth rate will correspond to the largest positive eigenvalue of $A$ in the finite dimensional case). Therefore, the effect of control on a mode which is unstable will be to temper its growth rate and "trim" it to the maximum value $\frac{r}{2}$.

\footnotetext{
${ }^{11}$ This is in fact equivalent to the assertion that the optimal path satisfies temporal transversality conditions at infinity.
} 
Remark 14 (The $\theta \rightarrow \infty$ limit). As seen by Proposition 9 in the $\theta \rightarrow \infty$ limit, the control has a stabilizing effect on unstable modes of the uncontrolled system.

Remark 15 (The $\theta \rightarrow 0$ limit). Similarly, by Proposition 9 in the $\theta \rightarrow 0$ limit, the robust control has a destabilizing effect on modes of the uncontrolled system which are "marginal" to be stable i.e. with $\hat{\alpha}_{k}$ negative but close to zero.

Remark 16 (Similarities and Differences with Turing instability). This is similar to Turing instability leading to pattern formation but with a very important difference! In contrast to Turing instability which is observed in an uncontrolled forward Cauchy problem, this instability is created in an optimally controlled problem in the infinite horizon. This has important consequences and repercussions both from the conceptual as well as from the practical point of view. On the conceptual level, a controlled system is related to a system that somehow its final state (at $t \rightarrow \infty$ in our case) is predescribed. Therefore, our result is an "extension" of Turing instability in a forward-backward system and not just to a forward Cauchy problem, as is the case for the Turing instability. On the practical point of view, the optimal control nature of the problem we study here induces serious constraints on the growth rate of the allowed patterns which has a strict upper bound is related only to the discount factor of the model and not on the operator $A$. This is not the case for the standard Turing pattern formation mechanism, in which the growth rate upper bound is simply related to the spectrum of the operator $A$.

\subsection{Hot spot of type III: The cost of robustness}

The value function is of the form $V_{k}=\frac{M_{2, k}}{2} \hat{x}_{k}^{2}+\frac{\hat{c}_{k}^{2} M_{2, k}}{2 r}$. This gives us the total cost of the minimum possible deviation from the desired goal and it is made up from contributions by three terms:

$\triangleright$ the term proportional to $p$ in the cost functional which corresponds to the cost related to the deviation from the desired target,

$\triangleright$ the term proportional to $q$ in the cost functional which corresponds to the cost related to the cost of the control $u$ needed to drive the system to the desired target and

$\triangleright$ the term proportional to $\theta$ in the cost functional which corresponds to the cost of robustness (which is the cost incurred by the regulator because she wants to be robust when she has concerns about the misspecification of the model).

The value functions depends on all these three contributions and this may be clearly seen since $M_{2, k}$ is in fact a function of the parameters $p, q, \theta$.

An interesting question is which is the relevant importance of each of these contributions in the overall value function. Does one term dominates over the others or not?

A simple answer to this question will be given by the elasticity of the value function with respect to these parameters, i.e., by the calculation of the quantities $\frac{1}{V} \frac{\partial V}{\partial p}, \frac{1}{V} \frac{\partial V}{\partial q}$ and $\frac{1}{V} \frac{\partial V}{\partial \theta}$. It is easily seen that these elasticities are independent of $\hat{x}_{k}$ and reduce to $\frac{1}{M_{2, k}} \frac{\partial M_{2, k}}{\partial p}, \frac{1}{M_{2, k}} \frac{\partial M_{2, k}}{\partial q}$ and $\frac{1}{M_{2, k}} \frac{\partial M_{2, k}}{\partial \theta}$, respectively. Whenever one of these quantities tends to infinity, that means that the contribution of the relevant procedure dominates the control problem ${ }^{12}$

In particular whenever $\frac{1}{M_{2, k}} \frac{\partial M_{2, k}}{\partial \theta} \rightarrow \infty$, then we say that the cost of robustness becomes more expensive than what it offers, and we will call that a hot spot of type III. This quantity can be

${ }^{12}$ This interpretation arises from observation that close to a point $\left(p_{0}, q_{0}, \theta_{0}\right)$ the value function behaves as

$$
\left.V_{k} \simeq \frac{\partial V_{k}}{\partial p}\right|_{p=p_{0}}\left(p-p_{0}\right)+\left.\frac{\partial V_{k}}{\partial q}\right|_{q=q_{0}}\left(q-q_{0}\right)+\left.\frac{\partial V_{k}}{\partial \theta}\right|_{\theta=\theta_{0}}\left(\theta-\theta_{0}\right) .
$$


calculated directly from the solution of the quadratic equation (13) through straightforward but tedious algebraic manipulations, which we choose not to reproduce here.

However, an illustrative partial case, which allows some insight on the nature of hot spots of type III is the following:

Differentiating (13) with respect to $\theta$ yields

$$
-\frac{\hat{c}_{k}^{2}}{2 \theta^{2}} M_{2, k}^{2}+2\left(\frac{\hat{c}_{k}^{2}}{2 \theta}-\frac{\hat{b}_{k}^{2}}{2 q}\right) M_{2, k} \frac{\partial M_{2, k}}{\partial \theta}+\left(2 \hat{a}_{k}-r\right) \frac{\partial M_{2, k}}{\partial \theta}=0 .
$$

Dividing by $M_{2, k}^{2}$ we obtain

$$
-\frac{\hat{c}_{k}^{2}}{2 \theta^{2}}+2\left(\frac{\hat{c}_{k}^{2}}{2 \theta}-\frac{\hat{b}_{k}^{2}}{2 q}\right) \frac{1}{M_{2, k}} \frac{\partial M_{2, k}}{\partial \theta}+\left(2 \hat{a}_{k}-r\right) \frac{1}{M_{2, k}^{2}} \frac{\partial M_{2, k}}{\partial \theta}=0 .
$$

Let us now take the particular case where $2 \hat{a}_{k}=r$, so that

$$
\frac{1}{M_{2, k}} \frac{\partial M_{2, k}}{\partial \theta}=\frac{\hat{c}_{k}^{2}}{4 \theta\left(\frac{\hat{c}_{k}^{2}}{2 \theta}-\frac{\hat{b}_{k}^{2}}{2 q}\right)}
$$

which becomes infinite for values of $\theta$ such that $\theta \rightarrow \frac{q \hat{c}_{k}^{2}}{\hat{b}_{k}^{2}}$. The general case $2 \hat{a}_{k} \neq r$ may present similar phenomena.

\subsection{Non translation invariant systems}

The methodology employed in this section to provide closed form solutions used the translation invariant property of the dynamical system, which allowed the use of the discrete Fourier transform. This is a symmetry property of the system (commutation of the vector field with the translation operator) which has as a result that the spatial operators are convolutions and therefore the discrete Fourier transform may be used to turn this convolution into a product in Fourier space. This situation may be generalized for other symmetry groups and may lead to interesting generalizations for systems which are not translation invariant but invariant under other more complicated symmetries. In this case the tools of harmonic analysis on groups (see e.g. Rudin (1990)) may be used and generalized Fourier transforms may be defined in terms of the Haar measure ${ }^{13}$. In terms of this generalized Fourier transform, the system will decouple thus allowing for use of the proposed method in more general settings (see e.g. Bamieh et al. (2002) for a related discussion).

\section{The general linear quadratic control problem}

We now relax the simplifying (and restrictive) assumptions concerning the translation invariance property of the operators $\mathrm{A}, \mathrm{B}, \mathrm{C}$ as well as the overly restrictive assumption that $\mathrm{P}=p I$ and $\mathrm{Q}=q I$.

We now consider instead the solution of the general linear quadratic robust control problem (5) under the state constraint (3), and comment on the possibility of hot spot formation working in real space directly rather than in Fourier transform space. The general form of the problem allows the study of a wider range of economic applications (see, e.g., Section 8 for an illustration of the applicability of the general problem). The relaxation of translation invariance leads to significant complications, and to the inability to derive solutions in closed form. However, as our subsequent analysis shows the qualitative aspects regarding hot spot formation in general linear quadratic problems persist, beyond the translation invariant case.

\footnotetext{
${ }^{13}$ The Haar measure is a generalization of the Lebesgue measure, which is invariant under the symmetry group
} 


\subsection{Solution in terms of the Riccati equation}

The problem may be treated using the Hamilton-Jacobi-Bellman-Isaacs equation, which is solvable in terms of an operator (matrix) Riccati equation.

Theorem 1. If the problem (5) under the dynamic constraint (3) has a solution, for arbitrary $x \in \mathbb{H}$, then the optimal controls are of the feedback control form

$$
u=-\mathrm{Q}^{-1} \mathrm{~B}^{*} \mathrm{H}^{s y m} x, \quad v=\frac{1}{\theta} \mathrm{R}^{-1} \mathrm{C}^{*} \mathrm{H}^{s y m} x,
$$

and the optimal state satisfies the Ornstein-Uhlenbeck equation

$$
d x=\left(\mathrm{A}-\mathrm{BQ}^{-1} \mathrm{~B}^{*} \mathrm{H}^{\text {sym }}+\frac{1}{\theta} \mathrm{CR}^{-1} \mathrm{C}^{*} \mathrm{H}^{\text {sym }}\right) x d t+\mathrm{C} d W
$$

where $\mathrm{H}^{\text {sym }}$ is the solution of the operator Riccati equation

$$
\mathrm{H}^{\text {sym }} \mathrm{A}+\mathrm{A}^{*} \mathrm{H}^{\text {sym }}-\mathrm{H}^{\text {sym }} \mathrm{E}^{\text {sym }} \mathrm{H}^{\text {sym }}-r \mathrm{H}^{\text {sym }}+\mathrm{P}=0
$$

and $\mathrm{E}^{\text {sym }}:=\frac{1}{2}\left(\mathrm{E}+\mathrm{E}^{*}\right)$ is the symmetric part of $\mathrm{E}:=\mathrm{BQ}^{-1} \mathrm{~B}^{*}-\frac{1}{\theta} \mathrm{CR}^{-1} \mathrm{C}^{*}$.

Proof: We first obtain the relevant Hamilton-Jacobi-Bellman-Isaacs equation for this stochastic differential game. The generator for the Ornstein-Uhlenbeck equation is of the form

$$
\mathcal{L} V=\sum_{n}\left(\sum_{m}\left(a_{n m} x_{m}+b_{n m} u_{m}+c_{n m} v_{m}\right)\right) \frac{\partial}{\partial x_{n}}+\sum_{n} \sum_{m} \sum_{k} c_{n k} c_{m k} \frac{\partial^{2}}{\partial x_{n} \partial x_{m}}
$$

or in compact form

$$
\mathcal{L} V=\langle\mathrm{A} x+\mathrm{B} u+\mathrm{C} v, D V\rangle+\operatorname{Tr}\left(\mathrm{CC}^{*} D^{2} V\right)
$$

where $D V$ is the finite sequence (in $\ell^{2}=\ell^{2}\left(\mathbb{Z}_{N}\right) \simeq \mathbb{R}^{N}$ ) with $(D V)_{n}=\frac{\partial}{\partial x_{n}} V$ and $D^{2} V$ is the operator which can be represented as the matrix with elements $\left(D^{2} V\right)_{n m}=\frac{\partial^{2}}{\partial x_{n} \partial x_{m}} V$. With $\operatorname{Tr}$ we denote the trace of the operator involved whereas with the superscript $t r$ we denote the transposition.

We now construct the Hamiltonian. We start with the function $H: \mathbb{R}^{N} \times \mathbb{R}^{N} \times \mathbb{R}^{N} \rightarrow \mathbb{R}$ defined as

$$
H(V ; x, u, v)=\mathcal{L} V+\langle\mathrm{P} x, x\rangle+\langle\mathrm{Q} u, u\rangle-\theta\langle\mathrm{R} v, v\rangle
$$

We need to obtain the upper Hamiltonian and lower Hamiltonians defined respectively as

$$
\bar{H}:=\sup _{u} \inf _{v} H(V ; x, u), \underline{H}:=\inf _{v} \sup _{u} H(V ; x, u) .
$$

Let us present in some relative detail the construction of $\underline{H}$. The maximization over $u$ is a quadratic optimization problem over the Hilbert space $\mathbb{R}^{N}$. The first order condition is easily calculated to be

$$
\frac{1}{2}\left(\mathrm{Q}+\mathrm{Q}^{*}\right) u=-\mathrm{B}^{*} D V
$$

where by the symmetry of $Q$ and the positive definite property is is seen that

$$
u=-\mathrm{Q}^{-1} \mathrm{~B}^{*} D V
$$

We work similarly with the minimization problem over $v$ which is again a quadratic optimization problem in $\mathbb{R}^{N}$ whose first order conditions yield

$$
\frac{\theta}{2}\left(\mathrm{R}^{*}+\mathrm{R}\right) v=\mathrm{C}^{*} D V
$$


which upon invoking the symmetry of the operator $\mathrm{R}$ and the positive definite property yields

$$
v=\frac{1}{\theta} \mathrm{R}^{-1} \mathrm{C}^{*} D V
$$

We now insert these expressions for $u$ and $v$ back into $H$ to obtain

$$
\begin{aligned}
\underline{H} & =\left\langle\mathrm{A} x-\mathrm{BQ}^{-1} \mathrm{~B}^{*} D V+\frac{1}{\theta} \mathrm{CR}^{-1} \mathrm{C}^{*} D V, D V\right\rangle+\operatorname{Tr}\left(\mathrm{CC}^{*} D^{2} V\right) \\
& +\frac{1}{2}\langle\mathrm{P} x, x\rangle+\frac{1}{2}\left\langle\mathrm{QQ}^{-1} \mathrm{~B}^{*} D V, \mathrm{Q}^{-1} \mathrm{~B}^{*} D V\right\rangle-\frac{1}{2 \theta}\left\langle\mathrm{RR}^{-1} \mathrm{C}^{*} D V, \mathrm{R}^{-1} \mathrm{C}^{*} D V\right\rangle
\end{aligned}
$$

which upon rearrangement yields

$$
\underline{H}=\langle\mathrm{A} x, D V\rangle-\frac{1}{2}\left\langle\left(\mathrm{BQ}^{-1} \mathrm{~B}^{*}-\frac{1}{\theta} \mathrm{CR}^{-1} \mathrm{C}^{*}\right) D V, D V\right\rangle+\frac{1}{2}\langle\mathrm{P} x, x\rangle+\operatorname{Tr}\left(\mathrm{CC}^{*} D^{2} V\right)
$$

The lower Hamilton-Jacobi-Bellman-Isaacs then becomes

$$
\langle\mathrm{A} x, D V\rangle-\frac{1}{2}\left\langle\left(\mathrm{BQ}^{-1} \mathrm{~B}^{*}-\frac{1}{\theta} \mathrm{CR}^{-1} \mathrm{C}^{*}\right) D V, D V\right\rangle+\frac{1}{2}\langle\mathrm{P} x, x\rangle+\frac{1}{2} \operatorname{Tr}\left(\mathrm{CC}^{*} D^{2} V\right)=r V
$$

This is a nonlinear elliptic equation in $\mathbb{R}^{N}$, the solution of which will provide the optimal controls.

On account of the quadratic nature of the problem we seek a solution of the following ansatz

$$
V(x)=\frac{1}{2}\langle\mathrm{H} x, x\rangle+\langle j, x\rangle+K
$$

where $\mathrm{H}$ is an operator, $j$ is an element of the Hilbert space $\mathbb{R}^{N}$ and $K \in \mathbb{R}$. For this choice ${ }^{14}$

$$
D V=\frac{1}{2}\left(\mathrm{H}+\mathrm{H}^{*}\right) x+j, \quad D^{2} V=\frac{1}{2}\left(\mathrm{H}+\mathrm{H}^{*}\right) .
$$

We now substitute into the HJBI equation and match powers of $x$.

(a) The quadratic terms yield the operator Riccati equation

$$
\frac{1}{2}\left(\mathrm{H}^{*}+\mathrm{H}\right) \mathrm{A}-\frac{1}{8}\left(\mathrm{H}^{*}+\mathrm{H}\right) \mathrm{E}\left(\mathrm{H}+\mathrm{H}^{*}\right)+\frac{1}{2}(\mathrm{P}-r \mathrm{H})=0
$$

where

$$
\mathrm{E}:=\mathrm{BQ} \mathrm{Q}^{-1} \mathrm{~B}^{*}-\frac{1}{\theta} \mathrm{CR} \mathrm{R}^{-1} \mathrm{C}^{*}
$$

the solution of which yields $\mathrm{H}$.

(b) The terms which are linear in $x$ provide a linear homogeneous equation for $j$ which admits the trivial solution $j=0$.

(c) Finally, the constant term $K$ is

$$
K=\frac{1}{2 r} \operatorname{Tr}\left(\mathrm{CC}^{*} \frac{\mathrm{H}+\mathrm{H}^{*}}{2}\right) .
$$

A quick inspection of the solution shows that we only need the symmetric part of the operator $\mathrm{H}$, $\mathrm{H}^{\text {sym }}:=\frac{1}{2}\left(\mathrm{H}+\mathrm{H}^{*}\right)$, in order to specify the optimal control and the value function ${ }^{15}$.

We therefore need to obtain a Riccati equation involving $\mathrm{H}^{\text {sym }}$ only.

\footnotetext{
${ }^{14} \mathrm{If} \mathrm{H}$ is a symmetric operator, then the above expressions simplify to $D V=\mathrm{H} x+j, D^{2} V=\mathrm{H}$, however, we do not make this assumption at this point.

${ }^{15} \mathrm{It}$ is easily seen that $\langle\mathbf{H} x, x\rangle=\left\langle\mathbf{H}^{s y m} x, x\right\rangle$.
} 
Taking the adjoint of equation (28) and adding we obtain the following symmetric Riccati equation for $\mathrm{H}^{\text {sym }}:=\frac{1}{2}\left(\mathrm{H}+\mathrm{H}^{*}\right)$ in the form

$$
\mathrm{H}^{\text {sym }} \mathrm{A}+\mathrm{A}^{*} \mathrm{H}^{\text {sym }}-\mathrm{H}^{\text {sym }} \mathrm{E}^{\text {sym }} \mathrm{H}^{\text {sym }}-r \mathrm{H}^{\text {sym }}+\mathrm{P}=0
$$

where $E^{s y m}:=\frac{1}{2}\left(E+E^{*}\right)$ is the symmetric part of $E$. In the above calculation we have explicitly taken into account the symmetry of $P$.

The determination of the optimal controls requires only the solution of the symmetric Riccati operator equation (29). Once $\mathbf{H}^{\text {sym }}$ is known the feedback control laws are given by

$$
u=-\mathrm{Q}^{-1} \mathrm{~B}^{*} \mathrm{H}^{s y m} x, \quad v=\frac{1}{\theta} \mathrm{R}^{-1} \mathrm{C}^{*} \mathrm{H}^{\text {sym }} x,
$$

where now $x$ is assumed to be the current state of the system.

Remark 17. The operator Riccati equation (29) is the generalization of the quadratic algebraic equation (13) in the case where the operators $A, B$ and $C$ are not translation invariant, and thus amenable to analysis using the Fourier transform. Furthermore, in the case where the state space is finite dimensional (i.e., in the case of finite lattices) the operator Riccati equation assumes the form of a matrix Riccati equation.

Clearly, by Theorem 1 the solvability and the properties of the solution for the optimal control problem is reduced to the solvability and the properties of the solution of the operator Riccati equation (29).

Proposition 10. Let $m=\|\mathrm{A}\|$ defined as $m=\left\{\sup \langle\mathrm{A} x, x\rangle,\|x\|_{\mathbb{R}^{N}}=1\right\}$ and assume that $m<r / 2$. Then, for small enough values of $\|\mathrm{E}\|$ and $\|\mathrm{P}\|$ the operator Riccati equation (29) admits a unique bounded strong solution.

Proof: By further defining the operator $\tilde{\mathrm{A}}=\mathrm{A}-\frac{r}{2} I$ the symmetric operator Riccati equation simplifies to

$$
\mathrm{H}^{\text {sym }} \tilde{\mathrm{A}}+\tilde{\mathrm{A}}^{\text {tr }} \mathrm{H}^{\text {sym }}-\mathrm{H}^{\text {sym }} \mathrm{E}^{\text {sym }} \mathrm{H}^{\text {sym }}+\mathrm{P}=0
$$

This is in the standard form of operator Riccati equation studied in the literature (see e.g., Bensoussan et al. (1992) or Da Prato (2002)). The spectrum of the operator $\tilde{\mathrm{A}}$ is in the interval $\left[-m-\frac{r}{2}, m-\frac{r}{2}\right]$ whereas the spectrum of the operator $-\tilde{\mathrm{A}}$ is in the interval $\left[\frac{r}{2}-m, m+\frac{r}{2}\right]$. If $m<\frac{r}{2}$ then $d:=$ $\operatorname{dist}(\operatorname{spec}(\tilde{\mathrm{A}}), \operatorname{spec}(-\tilde{\mathrm{A}}))>0$. Then according to Theorem 3.7 in Albeverio et al. (2003) (whose proof is based on the Banach contraction theorem) equation (30) has a unique solution.

Remark 18. The "smallness" condition on $\|E\|$ and $\|P\|$ is made explicit via the Banach contraction argument in the proof of Theorem 3.7 in Albeverio et al. (2003). In particular, for the existence of a strong solution we need $\|\mathrm{E}\|+\|\mathrm{P}\|<d$. It can be seen that this condition breaks down for small enough values of $\theta$, which in fact is the analogue of the hot spot of Type I that was obtained before. for the restricted class of models involving translation invariant operators, using the Fourier expansion method (see Proposition 7).

\subsection{Hot spot formation in general linear quadratic systems}

The various hot spots that were obtained explicitly for the translation invariance case, can be generalized for the general linear quadratic case.

Concerning hot spots of Type I, these are related to the breakdown of the minimax problem involved, for small values of the parameter $\theta$. In fact, in McMillan and Triggiani (1994) it has been shown rigorously for a similar deterministic problem that there does not exist a saddle point and the 
functional becomes unbounded if $\theta<\theta_{c r}$ where $\theta_{c r}$ is an intrinsic parameter of the system related to the data of the system. This result has been shown with the use of Lyusternik theory, and can be extended for the stochastic case. In fact, our results concerning the solvability of the relevant Riccati equation are pointing in this direction. Therefore, hot spots of Type I do exist in general linear quadratic systems, and are indeed related to model mispecification costs.

Similarly, hot spots of Type II will also exist. It can be seen that if the operators involved in the Riccati equation are diagonalizable then the Riccati equation admits a solution $\mathrm{H}^{\text {sym }}$ is a diagonalizable operator as well. Then, by the properties of diagonalizable operators the operators involved in the feedback laws are diagonalizable as well. A quick inspection shows that in this case the spectral theorem holds for the operator $\mathcal{R}$, therefore, the pattern formation behavior for the optimal path may now be obtained by spectral analysis. The hot spots of Type II will correspond to these eigenfunctions of the operator $\mathcal{R}:=\mathrm{A}-\mathrm{BQ}^{-1} \mathrm{~B}^{*} \mathrm{H}^{\text {sym }}+\frac{1}{\theta} \mathrm{CR}^{-1} \mathrm{C}^{*} \mathrm{H}^{\text {sym }}$ that have positive eigenvalues. A priori estimates of the spectrum may help us rule out the possibility of the emergence of hot spots.

The above general formulae for the feedback controls simplify in certain particular cases of interest. For instance, assume that we are interested in the localized entropic constraints problem introduced in Proposition 2. Then, since $\mathrm{R}$ is a diagonal operator, it can easily be seen that $\mathrm{R}^{-1}$ is also diagonal and has the representation $r_{n m}^{-1}=\theta_{n}^{-1} \delta_{n m}$. Therefore, the optimal feedback control $v$ can be expressed as

$$
v_{n}=\frac{1}{\theta_{n}}\left(\mathrm{C}^{*} \mathrm{H}^{s y m} x\right)_{n},
$$

where,

$$
\left(\mathrm{C}^{*} \mathrm{H}^{s y m} x\right)_{n}=\sum_{m} \mathrm{f}_{n m} x_{m}
$$

where $\mathrm{f}_{n m}$ are determined as long as the operators $\mathrm{C}^{*}, \mathrm{H}^{\text {sym }}$ are known. This form clarifies the effect of model misspecification in particular sites; the behavior of the system at lattice site $n$ depends inversely proportionally $\left(\frac{1}{\theta_{n}}\right.$ contribution) on the Lagrange multiplier of the localized entropic constraint at this site, as well as on the state of the system at neighboring sites through the term $\left(\mathrm{C}^{*} \mathrm{H}^{\text {sym }} x\right)_{n}$. This semi-explicit form allows us to understand the effect of model misspecification in certain sites. For instance sites with very large values of $\theta_{n}$ that in the absence of robust control tend to be unstable will remain so. Sites with small values of $\theta_{n}$, but larger than a critical cutoff value, that in the absence of model misspecification tend to be unstable may be stabilized as an effect of robustness. On the other hand extremely small values of $\theta_{n}$ may destabilize the system. This qualitative picture is in some sense a generalization of the arguments concerning hot spot formation, from the limited case of translation invariant operators to the general linear quadratic case.

The above remarks can help us understand the emergence of hot spots in the general linear model. Assume for simplicity that $\mathbf{C}$ is diagonal and that the spatial domain is finite so that $\theta=\left(\theta_{0}, \ldots, \theta_{N-1}\right)$ is the vector of local misspecification concerns. The low $\theta$ 's will correspond to locations with the higher concerns. If one or more of these low $\theta$ 's are such that the "smallness" condition on $\|\mathrm{E}\|$ and $\|\mathrm{P}\|$ is violated then local concerns will cause global regulation to break down.

In the same way if the low $\theta$ 's are such that the operator $\mathcal{R}$ has positive eigenvalues then local concerns may induce global spatial clustering through the mechanism described in section 4.2.

Pattern formation in the general linear quadratic system can also emerge through a 'non-Turing' mechanism. We can write the mean field for the optimal state as

$$
d x=\left[\mathrm{A}-\mathrm{BQ}^{-1} \mathrm{~B}^{*} \mathrm{H}^{\text {sym }}+\frac{1}{\theta} \mathrm{CR}^{-1} \mathrm{C}^{*} \mathrm{H}^{\text {sym }}\right] x=\mathcal{R} x
$$

Assume that matrix $A$ is invertible but matrix $\mathcal{R}$ which embodies optimization and misspecification concerns is not invertible. In this case the steady state equation $0=\mathcal{R} x$ will have more than one solutions. This means that there will be vectors $x \neq \mathbf{0}$ that will satisfy $0=\mathcal{R} x$. These vectors will be $\operatorname{ker}(\mathcal{R})$. If $\operatorname{ker}(\mathcal{R})$ consists of vectors which are spatially nonuniform then pattern formation emerges. 
This is pattern formation mechanism is however a non-Turing mechanism. Of course to examine whether such a mechanism exists a detailed analysis of matrix $\mathcal{R}$ and its null space is required. For example given the parametrization of the system one could ask the question whether a vector of misspecification concerns $\theta$ exists, such that $\mathcal{R}$ is not invertible. If such a vector exists then the specific misspecification concerns could induce pattern formation in the general linear quadratic model.

\section{Interlude: Linear quadratic approximation of nonlinear robust control problems}

The approach in sections 2-4 which provided the most tractable results, was based on linear quadratic problems. However, by using the linear quadratic approximation of general nonlinear control problems (see, e.g., Magill (1977a) ) we may adapt our results for the linear quadratic problem to obtain an approximation to a nonlinear robust optimal control problem. We sketch this approach.

Assume that we have a nonlinear problem, subject to weak additive noise, of the general form,

$$
d x_{n}=f\left(\sum_{m} a_{n m} x_{m}, \sum_{m} b_{n m} u_{m}\right) d t+\sum_{m} c_{n m} d w_{m} .
$$

The problem is subject to model uncertainty which may be modelled in terms of a drift $\{v\}$ so that applying Girsanov's theorem (see, e.g., Karatzas and Shreve (1991) for the finite dimensional theory or Carmona and Tehranchi (2006) for the case of infinite dimensions) in the same fashion as for the linear case we obtain the family of models

$$
d x_{n}=\left(f\left(\sum_{m} a_{n m} x_{m}, \sum_{m} b_{n m} u_{m}\right)+\epsilon \sum_{m} c_{n m} v_{m}\right) d t+\epsilon^{1 / 2} \sum_{m} c_{n m} d w_{m} .
$$

Assume now that the control $\{u\}$ has to be chosen so as to maximize a cost functional of the form

$$
J(u, v)=\max _{\{u\}} \min _{\{v\}} \mathbb{E}_{P}\left[\int_{0}^{\infty} e^{-r t} \sum_{n}\left(U\left(x_{n}(t), u_{n}(t)\right)+\theta v_{n}^{2}(t)\right)\right.
$$

which is a robust control problem of maximizing the utility function for the system for the worst possible model.

Since the noise is assumed to be weak we may consider as a zeroth order approximation a deterministic optimal path which is uniform in space, i.e. a solution $\left\{x_{n}^{0}(t)\right\}$ such that $x_{n}^{0}(t)=x^{0}(t)$ for all $n \in \mathbb{Z}$. This is the solution of a deterministic optimal control problem, which corresponds to the minimization of $J(u, v)$ for the unperturbed (deterministic) state equation (33) with $\epsilon=0$ and is supported by a uniform in space control $\left\{u^{0}\right\}$ and uncertainty drift $\left\{v^{0}\right\}$. Let us consider perturbations of $\{x, u, v\}$ around this reference solution, i.e. let us consider solutions of the above problem of the form

$$
\{x, u, v\}=\left\{x^{0}, u^{0}, v^{0}\right\}+\epsilon\left\{x^{1}, u^{1}, v^{1}\right\}
$$

where now $\{x, u, v\}$ are subject to uncertainty and are solutions of the stochastic state equation (33) with $\epsilon$ a small parameter. The perturbation is assumed to be spatially dependent.

We linearize the state equation around the state $\left\{x^{0}, u^{0}, v^{0}\right\}$ to obtain to first order in $\epsilon$ that

$$
d x_{n}^{1}=\left(A \sum_{m} a_{n m} x_{m}^{1}+B \sum_{m} b_{n m} u_{m}^{1}+\sum_{m} c_{n m} v_{m}^{1}\right) d t+\sum_{m} c_{n m} d w_{m}
$$

where

$$
A:=f_{1}\left(\sum_{m} a_{n m} x_{m}^{0}(t), \sum_{m} b_{n m} u_{m}^{0}(t)\right), \quad B:=f_{2}\left(\sum_{m} a_{n m} x_{m}^{0}(t), \sum_{m} b_{n m} u_{m}^{0}(t)\right)
$$


where $f_{1}, f_{2}$ are the partial derivatives of $f$ with respect to the first and the second variable respectively. In general $A$ and $B$ are functions of time but not of space. In the special cases where either $\left\{x^{0}, u^{0}\right\}$ are steady states or the operators generated by the matrices $\left\{a_{n m}\right\},\left\{b_{n m}\right\}$ are such that $\sum_{m} a_{n m}=0$, $\sum_{m} b_{n m}=0$ (diffusive coupling) the functions $A$ and $B$ are constant.

We furthermore look at the local behavior of the cost functional $J(u, v)$ around the state $\left\{x^{0}, u^{0}\right\}$. To be more precise, we calculate $J\left(u^{0}+\epsilon u^{1}, v^{0}+\epsilon v^{1}\right)$ and Taylor expand in $\epsilon$. The first order term in the expansion is effectively the Gâteaux derivative of the functional $J$ calculated at $\left\{u^{0}, v^{0}\right\}$, and by the extremality properties of $\left\{x^{0}, u^{0}, v^{0}\right\}$ this vanishes. We are thus left with the second order terms in this expansion which are

$$
J^{1}=\int_{0}^{\infty} e^{-r t} \sum_{n}\left(U_{11}\left(x_{n}^{1}(t)\right)^{2}+U_{22}\left(u_{n}^{1}(t)\right)^{2}-|\theta|\left(v_{n}^{1}(t)\right)^{2} d t\right.
$$

where $U_{i j}, i, j=1,2$ are the second derivatives of the utility function $U$ with respect to the first and second variable calculated at $\left(\sum_{m} a_{n m}\right) x^{0}(t)$ and $\left(\sum_{m} b_{n m}\right) u^{0}(t)$. Therefore, $U_{i j}=U_{i j}(t)$ are deterministic functions of time (but not of space). If the utility function $U$ is separable then $U_{12}=0$. We may assume this without loss of generality.

The above discussion shows (rather informally) that the problem of extremizing $J(u, v)$ subject to the stochastic state equation (33) may be approximated by the problem of extremizing $J^{1}\left(u^{1}, v^{1}\right)$ subject to the linear state equation (35). This is a linear quadratic control problem similar to the one studied here. If $x^{0}, u^{0}, v^{0}$ are time independent (steady states) then this linear quadratic control problem is of the exact form studied here and the results of this paper may be used in their exact form to study the approximation of the nonlinear problem. If $x^{0}, u^{0}, v^{0}$ are time dependent then the linear quadratic control problem is one with time varying deterministic coefficients, which is still manageable by a slight modification of the results of this paper.

Remark 19. The above linear quadratic approximation to a nonlinear problem may also have an alternative interpretation as follows: Consider any desired path $\left\{x^{0}(t)\right\}$ and study small deviations from that. Linearizing the state equation we obtain a linear system similar to (35). Then the problem is to pick the controls $u$ so as to keep the perturbed problem (35) as close as possible to the desired target $\left\{x^{0}(t)\right\}$,for the worst case scenario in terms of a whole family of models (specified by $\{v\}$ ). If the distance from the target is given by a quadratic distance functional, and the model misspecification is given by an entropic measure then it is easy to see that the above mentioned "stabilization" problem is equivalent to a linear quadratic robust control problem of the form treated in this paper.

\section{Nonlinear systems}

\subsection{General form of the controlled system}

Consider now the nonlinear system

$$
d x=(\mathrm{A} x+\mathrm{F}(x)+\mathrm{B} u) d t+\mathrm{C} d w
$$

where as before $x \in \mathbb{R}^{N}, \mathrm{~A}: \mathbb{R}^{N} \rightarrow \mathbb{R}^{N}$ is a linear operator and $\mathrm{F}: \mathbb{R}^{N} \rightarrow \mathbb{R}^{N}$ is in general a nonlinear operator and $C$ is the covariance operator. The simplest choice for the nonlinear term $\mathrm{F}$ may be $\mathbf{F}(x)=\left(f_{1}\left(x_{1}\right), f_{2}\left(x_{2}\right), \cdots\right)$ in which case the nonlinear effects are purely local, however this is by no means a necessary restriction ${ }^{16}$. The functions $\left\{f_{i}\right\}$ will be assumed to be twice differentiable, together with their derivatives and satisfying dissipativity conditions. The control acts on the system through the linear operator $\mathrm{B}: \mathbb{R}^{N} \rightarrow \mathbb{R}^{N}$.

\footnotetext{
${ }^{16}$ The general form $\mathrm{F}(x)=\left(f_{1}(x), f_{2}(x), \cdots\right)$ where at each site the nonlinear effects depends on the state of the system at all lattice sites is easily handled within the framework presented here.
} 
The robust form of the system, using the Girsanov theorem is

$$
d x=(\mathrm{A} x+\mathrm{F}(x)+\mathrm{B} u+\mathrm{C} v) d t+\mathrm{C} d w .
$$

We now consider a control functional of the form

$$
J=\mathbb{E}_{Q}\left[\int_{0}^{\infty} e^{-r t}(\mathrm{U}(x(t))+\mathrm{K}(u(t))-\mathrm{T}(v(t))) d t\right]
$$

where $\mathrm{U}: \mathbb{R}^{N} \rightarrow \mathbb{R}$ is a measure of distance from a desired target, $\mathrm{K}: \mathbb{R}^{N} \rightarrow \mathbb{R}$ is a cost function for the control and $\mathrm{T}: \mathbb{R}^{N} \rightarrow \mathbb{R}$ is a cost function for the robustness. All three functions are assumed convex. The robust control problem thus becomes

$$
\min _{u} \max _{v} \mathbb{E}_{Q}\left[\int_{0}^{\infty} e^{-r t}(\mathrm{U}(x(t))+\mathrm{K}(u(t))-\mathrm{T}(v(t))) d t\right]
$$

subject to the nonlinear state equation (36). By $\mathrm{K}^{£}: \mathbb{H}^{*} \rightarrow \mathbb{R}$ we denote the Fenchel-Legendre transform of $\mathrm{K}$ defined by

$$
\mathrm{K}^{£}(p):=\sup _{x \in \mathbb{H}}[\langle p, x\rangle-\mathrm{K}(x)]
$$

where by the Riesz representation we assume that the dual space $\mathbb{H}^{*} \simeq \mathbb{H}^{17}$.

\subsection{Solution in terms of the HJBI equation}

The nonlinear optimal control problem may be treated in terms of a fully nonlinear Hamilton-JacobiBellman-Isaacs equation.

Theorem 2. The Hamilton-Jacobi-Bellman-Isaacs equation associated with the robust control problem (37) subject to the constraint (36) is the nonlinear PDE

$$
\langle\mathrm{A} x+\mathrm{F}(x), D V\rangle+\operatorname{Tr}\left(\mathrm{CC}^{*} D^{2} V\right)+\mathrm{U}(x)-\mathrm{K}^{£}\left(-\mathrm{B}^{*} D V\right)+\mathrm{T}^{£}\left(\mathrm{C}^{*} D V\right)=r V
$$

where $\mathrm{K}^{£}, \mathrm{~T}^{£}$ are the Fenchel-Legendre transforms of $\mathrm{K}$ and $\mathrm{T}^{£}$ respectively. Given a solution of this equation $V: \mathbb{H} \rightarrow \mathbb{R}$ of sufficient regularity the associated closed loop system is the nonlinear Ornstein-Uhlenbeck system

$$
d x=\left(\mathrm{A} x+\mathrm{F}(x)-D \mathrm{~K}^{£}\left(\mathrm{~B}^{*} D V(x)\right)+D \mathrm{~T}^{£}\left(\mathrm{C}^{*} D V(x)\right)\right) d t+\mathrm{C} d w
$$

Proof: The generator operator for the diffusion process defined by the solution of (36) is the linear operator $\mathcal{L}$ whose action on a suitably smooth function $\Phi$ is given by

$$
\mathcal{L} \Phi=\sum_{n}\left(\sum_{m}\left(a_{n m} x_{m}+b_{n m} u_{m}+c_{n m} v_{m}\right)+f_{n}\left(x_{n}\right)\right) \frac{\partial}{\partial x_{n}} \Phi+\sum_{n} \sum_{m} \sum_{k} c_{n k} c_{m k} \frac{\partial^{2}}{\partial x_{n} \partial x_{m}} \Phi
$$

or in compact form

$$
\mathcal{L} \Phi=\langle\mathrm{A} x+\mathrm{F}(x)+\mathrm{B} u+\mathrm{C} v, D \Phi\rangle+\operatorname{Tr}\left(\mathrm{CC}^{*} D^{2} \Phi\right)
$$

We now consider the Hamiltonian

$$
H(V ; x, u, v)=\mathcal{L} V+\mathrm{U}(x)+\mathrm{K}(u)+\mathrm{T}(v),
$$

\footnotetext{
${ }^{17}$ In this particular case $\mathbb{H}=\ell^{2}:=\ell^{2}\left(\mathbb{Z}_{N}\right) \simeq \mathbb{R}^{N}$ and by the finite dimensionality of the Hilbert space involved $\mathbb{H}=\mathbb{H}^{*}$.
} 
which may be rewritten as a sum of three terms

$$
\begin{aligned}
& H(V ; x, u, v)=H_{1}(V ; x)+H_{2}(V ; u)+H_{3}(V ; v), \\
& H_{1}(V ; x):=\langle\mathrm{A} x+\mathrm{F}(x), D V\rangle+\operatorname{Tr}\left(\mathrm{CC}^{*} D^{2} V\right)+\mathrm{U}(x), \\
& H_{2}(V ; u):=\langle\mathrm{B} u, D V\rangle+\mathrm{K}(u), \\
& H_{3}(V ; v):=\langle\mathrm{C} v, D V\rangle+\mathrm{T}(v),
\end{aligned}
$$

Note that

$$
\begin{aligned}
\inf _{u} H_{2}(V ; u) & =\inf _{u}(\langle\mathrm{~B} u, D V\rangle+\mathrm{K}(u))=\inf _{u}\left(\left\langle u, \mathrm{~B}^{*} D V\right\rangle+\mathrm{K}(u)\right) \\
& =-\sup _{u}\left(\left\langle u,\left(-\mathrm{B}^{*} D V\right)\right\rangle-\mathrm{K}(u)\right)=-\mathrm{K}^{£}\left(-B^{t r} D V\right),
\end{aligned}
$$

where $\mathrm{K}^{£}$ denotes the Fenchel-Legendre transform of $\mathrm{K}$. By the theory of the Fenchel-Legendre transform (see e.g., Aubin and Ekeland (1984)) $p \in \partial \mathrm{K}(u)$ is equivalent to $u \in \partial \mathrm{K}^{£}(p)$ where $\partial$ denotes the subdifferential operator, therefore, by the regularity assumptions imposed on $\mathrm{K}$, the minimizer is

$$
u=-D \mathrm{~K}^{£}\left(\mathrm{~B}^{*} D V\right)
$$

Similarly,

$$
\sup _{v} H_{2}(V ; v)=\sup _{v}(\langle\mathrm{C} u, D V\rangle-\mathrm{T}(u))=\mathrm{T}^{£}\left(\mathrm{C}^{*} D V\right)
$$

where $\mathrm{T}^{£}$ is the Fenchel-Legendre transform of $\mathrm{T}$. By similar arguments as above, the maximizer is

$$
v=D \mathrm{~T}^{£}\left(\mathrm{C}^{*} D V\right)
$$

The Hamilton-Jacobi-Bellman-Isaacs equation assumes the form

$$
\langle\mathrm{A} x+\mathrm{F}(x), D V\rangle+\operatorname{Tr}\left(\mathrm{CC}^{*} D^{2} V\right)+\mathrm{U}(x)-\mathrm{K}^{£}\left(-\mathrm{B}^{*} D V\right)+\mathrm{T}^{£}\left(\mathrm{C}^{*} D V\right)=r V
$$

which is an infinite dimensional second order fully nonlinear partial differential equation for the value function $V$. The nonlinearity enters through the Fenchel-Legendre transforms $\mathrm{K}^{£}$ and $\mathrm{T}^{£}$ of $\mathrm{K}$ and $\mathrm{T}$ respectively. Assume the existence of a regular enough solution and substitute (41) and (43) into (36) to obtain the closed loop control system (39).

The solvability of the HBJI equation follows by generalizing results either of Da Prato and Zabczyk (2002) or Cerrai (2001), for stochastic control problems, to the case of stochastic differential games. We do not provide the detailed proof here for the sake of brevity. The proof uses techniques from the theory of maximal dissipative operators.

\subsection{Hot spot formation in nonlinear systems}

We now consider the possibility of hot spot formation in the nonlinear robust control system. Let us assume a steady state $x_{0} \in \mathbb{H}$ for the averaged over all realizations of the noise closed loop system, which presents no spatial patterns. This means that $x_{0}$ is such that $\mathrm{A} x_{0}=0$ and furthermore

$$
0=\mathrm{F}\left(x_{0}\right)-D \mathrm{~K}^{£}\left(\mathrm{~B}^{*} D V\left(x_{0}\right)\right)+D \mathrm{~T}^{£}\left(\mathrm{C}^{*} D V\left(x_{0}\right)\right)
$$

for a sufficiently smooth solution of the HJBI equation. We now consider a small perturbation of $x_{0}$ in the form $x=x_{0}+\epsilon z$ where $\epsilon$ is a small real number and $z \in \mathbb{H}$, and see how the closed loop system (39) evolves under this perturbation. A relevant question is whether $z$ develops any spatial variability which is interpreted as a hot spot of type II.

The following proposition provides some answer to this question. 
Proposition 11. Assume that $V$ is a $C^{2}$ solution of (38). If $\mathrm{K}^{£}$ and $\mathrm{T}^{£}$ are $C^{2}$ then the perturbation $z$ is the solution of the linear Ornstein-Uhlenbeck equation

$$
d z=\left(\mathrm{A} z+D \mathrm{~F}\left(x_{0}\right) z-D^{2} \mathrm{~K}^{£} \mathrm{~B}^{*} D^{2} V\left(x_{0}\right) z+D^{2} \mathrm{~T}^{£} \mathrm{C}^{*} D^{2} V\left(x_{0}\right) z\right) d t+\mathrm{C} d w
$$

The type II hot spots correspond to the unstable modes of the above equation, i.e., to eigenvectors of the matrix

$$
\mathcal{R}:=\mathrm{A}+D \mathrm{~F}\left(x_{0}\right)-D^{2} \mathrm{~K}^{£} \mathrm{~B}^{*} D^{2} V\left(x_{0}\right)+D^{2} \mathrm{~T}^{£} \mathrm{C}^{*} D^{2} V\left(x_{0}\right)
$$

with positive eigenvalues.

Proof: Substitute $x=x_{0}+\epsilon z$ into (39) and expand in powers of $\epsilon$, using the Taylor expansion theorem. According to that

$$
D V\left(x_{0}+\epsilon z\right)=D V\left(x_{0}\right)+\epsilon D^{2} V z+O\left(\epsilon^{2}\right)
$$

where $D^{2} V: \mathbb{H} \rightarrow \mathbb{H}$ is a symmetric operator corresponding to the generalization of the Hessian matrix. Therefore, by the assumed regularity of $\mathrm{K}^{£}$

$$
D \mathrm{~K}^{£}\left(\mathrm{~B}^{*} D V(x)\right)=D \mathrm{~K}^{£}\left(\mathrm{~B}^{*} D V\left(x_{0}\right)\right)+\epsilon D^{2} \mathrm{~K}^{£} \mathrm{~B}^{*} \epsilon D^{2} V z+O\left(\epsilon^{2}\right)
$$

and similarly for the other nonlinear terms. Inserting into the closed loop system yields the required result for the evolution of $z$. The rest follows by spectral theory considerations.

The value functions and the Legendre-Fenchel transforms satisfy convexity properties. This gives important information on the second derivatives $D^{2} \mathrm{~K}^{£}, D^{2} V\left(x_{0}\right), D^{2} \mathrm{~T}^{£}$ and in particular assuming sufficient regularity they are positive operators. This property allows us at least to obtain some a priori estimates on the spectrum of $\mathcal{R}$ and thus provide values on the parameters of the model which allow the generation of hot spot. In this respect, we may generalize some of the findings of the linear model in the nonlinear model.

\section{Application: Distance-dependent utility and robust control of in situ consumption}

An issue that has been given attention in spatial models of individual behavior is the concept of distance-dependent utility. In models of travel behavior the impact of distance on trip preferences underlies the choice of an individual to consume at locations which are away from his/her current location. The distance-dependent utility relates to the concept of spatial discounting which, similar to time discounting, provides weights which an individual attaches to utility derived at locations away from current location (e.g. Smith (1975), Smith (1976), Perrings and Hannon (2001), Wu and Plantinga (2003), Akamatsu et al. (2009)). For exponential spatial discounting for example, a spatial discount factor can be defined as $\alpha(n)=\beta^{-n}, \beta>1, n=0,1,2, \ldots, N-1$ indicating that the individual will attach declining weights to utility accruing at locations further away from his/her present location at $n=0$.

Spatial discounting and distance - dependent utility can be interpreted in terms of an individual expressing preferences for consuming at different points in space. This interpretation can be associated with traveling to consume for example environmental amenities which take the form of services generated by stocks of natural capital. According to the Millennium Ecosystem Assessment classification stocks of natural capital accumulated in ecosystems generate supporting, provisioning, regulating, and cultural services. Some of these services can be consumed only in situ which means that an individual needs to travel a certain distance in order to consume. Recreational or tourism related services is a classic example of in situ consumption. 
The analytical framework developed in this paper can be readily used to study the structure of equilibrium when utility is distance dependent and an individual consumes in locations away from her under uncertainty. We formulate this application in the context of an economy located on a discrete lattice defined in terms of a finite group of integers modulo $N$. That is in our spatial economy each location or each cell belongs to a discrete ring of cells with the property that the cell 1 is the same as cell $N$, cell 2 as cell $N+1$ and so. A representative consumer is located at each cell (location) $n=0,1 \ldots . N-1$. Each cell is characterized by a stock of natural capital $x_{n}(t)$ which generates environmental services that can be consumed only in situ.

Consumption at location $n$ is the sum of consumption of all individuals or $u_{n}(t)=\sum_{m=0}^{N-1} u_{n m}(t)$, where $u_{n m}(t)$ is the consumption of an individual located at location $m=0,1, \ldots N-1$ of services at location $n$. Consumption of services implies reduction of natural stocks. The evolution of the natural capital stock at a given location is determined by natural growth at the location and by the impact that stock levels at nearby locations might have on this natural growth rate. This impact might be positive or negative in the context of facilitating or competing growth. The evolution of the natural stock is subject to stochastic shocks. Thus we write

$$
d x_{n}(t)=\sum_{m=0}^{N-1}\left[\alpha_{n m} x_{m}(t)-\gamma_{n m} u_{n}(t)\right] d t+\sum_{m=0}^{N-1} c_{n m}(t) d w_{m},
$$

for the evolution of the stock of natural capital at location $n=0,1, \cdots, N-1$. In this formulation $x_{n}(t)$ for all $n$, can be interpreted as deviations from a spatially homogeneous benchmark equilibrium stock, $\bar{x}$ which could be determined historically (e.g. a preindustrial level). The terms $\alpha_{n m} x_{m}(t)$ of (44) can be regarded as a first order approximation around the benchmark steady state, with $a_{n n}$ being the value of the derivative of the first order approximation evaluated at the benchmark equilibrium. ${ }^{18}$. The influence kernel $\alpha_{n m}, n \neq m$ describes the spatial effects of nearby stocks on the growth of stock at $n$, while the influence kernel $\gamma_{n m}$ describes the spatial effects that consumption of services in nearby locations might have on the stock of amenities at location $n$. Influence kernels are assumed symmetric so the impact depends only on the distance between $n$ and $m$. Thus $\alpha_{n m} \equiv \alpha_{n-m}=\alpha_{m-n} \equiv \alpha_{m n}$, $\gamma_{n m} \equiv \gamma_{n-m}=\gamma_{m-n} \equiv \gamma_{m n}$.

\section{Consumers}

A representative individual located at a given location (cell) $n$ can derive utility by travelling to the other locations of the ring and consuming the corresponding amenity services. Let $\left(u_{n m}(t), b_{n m}(t)\right)$, $m, n=0,1, \ldots, N-1$, denote the consumption at location $m$ of an individual located at $n$ and the corresponding bliss point for the same individual. An individual located at point $n$ can travel to locations $0,1, \ldots, n-1, n+1, \ldots N-1$ to consume the services there and compare consumption to his/her corresponding bliss point.

We define individual utility in terms of square deviations of consumption from the bliss point or $U_{n}=\sum_{m} \beta_{n m}\left(u_{n m}(t)-b_{n m}(t)\right)^{2}, m=0, \ldots, N-1$, with the objective being the minimization of deviations from the corresponding bliss point (e.g. Ljungqvist and Sargent (2004)). The influence kernel $\beta_{n m}$ reflects the weight that the individual located at $n$, attaches to the utility derived at location $m$, so it can be interpreted as spatial discounting. We assume that the kernel is symmetric and that the impact depends only on the distance between $n$ and $m$ so that $\beta_{n m} \equiv \beta_{n-m}=\beta_{m-n} \equiv \beta_{m n}$. If $\beta_{n m}=\beta$ then all locations are treated equally. To consume services at location $m$ an individual located at $n$ should pay a known exogenous price $p_{m}(t) \geq 0$, which could be for example an entrance fee.

\footnotetext{
${ }^{18}$ The full nonlinear model can be considered in the context of Section 7 with similar qualitative result. The linear approach provides however better tractability.
} 
Assume that the individual has a quasi linear utility function with respect to a numeraire commodity, and that the price $p_{m}(t)$ is treated as parametric. The individual's problem is

$$
\max _{\left\{u_{n m}\right\}}-\sum_{m=0}^{n-1} \beta_{n m}\left(u_{n m}(t)-b_{n m}(t)\right)^{2}+I_{n}(t)-\sum_{m=0}^{N-1} p_{m}(t) u_{n m}(t) \text { for all } n
$$

where $I_{n}(t)$ is income at $n$, and by $\left\{u_{n m}\right\}$ we denote the whole family of processes $u_{n m}(\cdot)$. The solution results in individual demand curves for consumption at each location

$$
2 \beta_{n m} b_{n m}(t)-2 \beta_{n m} u_{n m}(t)=p_{m}(t)
$$

The aggregate demand at location $m$ and time $t, u_{n}(t):=\sum_{n=0}^{N-1} u_{n m}(t)$, is obtained as:

$$
u_{m}(t)=\sum_{n=0}^{N-1} b_{n m}(t)-\left(\sum_{n=0}^{N-1} \frac{1}{2 \beta_{n m}}\right) p_{m}(t)=: B_{0 m}(t)-B_{1 m}(t) p_{m}(t)
$$

where we have introduced the notation

$$
B_{0 m}(t):=\sum_{n=0}^{N-1} b_{n m}(t), B_{1 m}(t):=\left(\sum_{n=0}^{N-1} \frac{1}{2 \beta_{n m}}\right) .
$$

\section{The Regulator}

Consider now a regulator who seeks to allocate consumption at each location (cell) of the ring by maximizing utility across the whole spatial domain subject to the dynamic of the natural stocks. The regulator is concerned about possible misspecification of the dynamics. Allowing for uncertainty about the 'true' statistical distribution of the stocks, and following the discussion in Section 2, the evolution of the natural stocks can be written as:

$$
d x_{n}(t)=\sum_{m=0}^{N-1}\left[\alpha_{n m} x_{m}(t)-\gamma_{n m} u_{m}(t)+c_{n m} v_{m}(t)\right] d t+\sum_{m=0}^{N-1} c_{n m}(t) d w_{m}
$$

or in compact form as

$$
d x=(\mathrm{A} x+\Gamma u+\mathrm{C} v) d t+\mathrm{C} d w
$$

with the operators A, Г, C defined accordingly (and in particular represented as the matrices $\left\{a_{n m}\right\}$, $\left\{\gamma_{n m}\right\}$ and $\left.\left\{c_{n m}\right\}\right)$.

The robust control problem for the regulator becomes:

$$
\min _{\left\{u_{n m}(\cdot)\right\}} \max _{\left\{v_{n}(\cdot)\right\}} \mathbb{E}_{P}\left[\int_{0}^{\infty} e^{-r t}\left[\sum_{n=0}^{N-1} \sum_{m=0}^{N-1} \beta_{n m}\left(u_{n m}(t)-b_{n m}(t)\right)^{2}-\frac{\theta}{2}\left(v_{n}(t)\right)^{2}\right] d t\right]
$$

subject to (47)

A simplification of the problem that leads to closed form solutions is to assume that the regulator imposes an arbitrary bliss point $b_{n}(t)$ at each location and ignores the spatial weighting of the consumers by attaching the same weight $\beta=\beta_{n m}$ to all locations. In this case the regulators seeks that aggregate consumption at each location and solves the problem:

$$
\begin{aligned}
& \min _{\left\{u_{n}(\cdot)\right\}} \max _{\left\{v_{n}(\cdot)\right\}} \mathbb{E}_{P}\left[\int_{0}^{\infty} e^{-r t}\left[\sum_{n=0}^{N-1} \beta\left(u_{n}(t)-b_{n}(t)\right)^{2}-\frac{\theta}{2}\left(v_{n}(t)\right)^{2}\right] d t\right] \\
& \text { subject to (47) }
\end{aligned}
$$


Define the new control variable $\bar{u}=\left\{\bar{u}_{n}\right\}, \bar{u}_{n}=u_{n}-b_{n}$ and rewrite the regulator problem as

$$
\min _{\left\{u_{n}(\cdot)\right\}} \max _{\left\{v_{n}(\cdot)\right\}} \mathbb{E}_{P}\left[\int_{0}^{\infty} e^{-r t} \sum_{n=0}^{N-1} \beta\left(\bar{u}_{n}^{2}(t)-\frac{\theta}{2}\left(v_{n}(t)\right)^{2}\right] d t\right]
$$

subject to

$$
d x=(\mathrm{A} x+\Gamma \bar{u}+\mathrm{C} v+F) d t+\mathrm{C} d w
$$

where $b=\left\{b_{n}\right\}$, the bliss points at various lattice sites and $F=\Gamma b$ is known.

This is the simplified problem ${ }^{19}$, involving translation invariant operators, solved in Section 3, which in the Fourier space (Pontryagin dual space) is given by a problem similar to (12) treated in Proposition 5. Following the solution approach in Section 3, and taking without loss of generality ${ }^{20}$ the bliss points $b_{n}(t)=0$, we find that the feedback laws are

$$
\hat{u}_{k}^{*}=-\frac{\hat{\gamma}_{k} M_{2, k}}{2 \beta} \hat{x}_{k}^{*}, \quad \hat{v}_{k}^{*}=\frac{\hat{c}_{k} M_{2, k}}{2 \theta} \hat{x}_{k}^{*}
$$

where $M_{2, k}$ is the solution of the quadratic equation

$$
\left(\frac{\hat{c}_{k}^{2}}{2 \theta}-\frac{\hat{b}_{k}^{2}}{2 \beta}\right) M_{2, k}^{2}+\left(2 \hat{a}_{k}-r\right) M_{2, k}=0 .
$$

The expectation of $x$ evolves as

$$
d \hat{x}_{k}^{*}=R_{k} \hat{x}_{k}^{*} d t, \quad R_{k}=\hat{\alpha}_{k}-\frac{\hat{\gamma}_{k} M_{2, k}}{2 \beta}+\frac{\hat{c}_{k} M_{2, k}}{2 \theta}
$$

with solution

$$
\hat{x}_{k}^{*}(t)=\mathcal{A}_{k} \exp \left(R_{k} t\right), \hat{x}_{k}^{*}(0)=\hat{x}_{k 0}^{*} .
$$

Using the inverse Fourier transform we may obtain the optimal path and the optimal control in real space $^{21}$ as

$$
\begin{aligned}
& x_{n}^{*}(t)=\sum_{k=0}^{N-1} \mathcal{A}_{k} \exp \left[\frac{2 \pi i \omega z}{N}+R_{k} t\right] \\
& u_{n}^{*}(t)=\sum_{k=0}^{N-1} \frac{\hat{\gamma}_{k} M_{2, k}}{2 \beta} x_{n}^{*}(t) .
\end{aligned}
$$

and therefore the optimal supply of services at location $n$ is fully determined by (49). Then the equilibrium prices at locations $n$ will be determined by (46) as:

$$
p_{m}^{*}(t)=B_{0 m}(t)-\frac{u_{m}^{*}(t)}{B_{1 m}(t)}=B_{0 m}(t)-\frac{1}{B_{1 m}(t)}\left(\sum_{k=0}^{N-1} \frac{\hat{\gamma}_{k} M_{2, k}}{2 \beta} x_{n}^{*}(t)\right) .
$$

It should be noted that through (49) the local equilibrium prices depend on the local resource stock.

A more realistic model will allow for a differentiation of the weights across locations which would follow the preferences of the individuals' as well as a corresponding differentiation of the bliss points

\footnotetext{
${ }^{19}$ Apart from the introduction of the constant term $F$, which as can easily be seen does not alter the dynamics

${ }^{20}$ The case $b_{n}(t) \neq 0$ can be treated with minor modifications.

${ }^{21}$ In the primal lattice $\mathbb{Z}_{N}$ as opposed to the Fourier space which is the dual lattice.
} 
and site dependent misspecification concerns. In this case the regulator solves (48) subject to (47), which in a compact form, following the notation of Section 5 can be written as:

$$
\begin{aligned}
& \min _{u} \max _{v} \mathbb{E}_{P}\left[\int_{0}^{\infty} e^{-r t} \sum_{n=0}^{N-1}\left[\langle(\mathrm{~B} U)(t), U(t)\rangle-\theta_{n}\langle(\mathrm{R} v)(t)(v(t))\rangle\right] d t\right] \\
& \text { subject to } \\
& d x=(\mathrm{A} x+\Gamma u+\mathrm{C} v) d t+\mathrm{C} d w .
\end{aligned}
$$

where $U(t)=u-b$. This is the general linear quadratic control problem analyzed in Section 5 . Assuming without loss of generality ${ }^{22}$ that the bliss point is $b=0$, the optimal supply of services for an individual located at $m$ and consuming at $n$ will be

$$
u_{n m}^{*}(t)=-\mathrm{B}^{-1} \Gamma^{*} \mathrm{H}^{\mathrm{sym}} x_{n}(t)
$$

and the local equilibrium price at $n$ will be

$$
p_{n}^{*}(t)=B_{0 n}(t)-\frac{1}{B_{1 n}(t)}\left(\sum_{m=0}^{N-1} u_{n m}^{*}(t)\right) .
$$

By determining the supply of optimal services at each location the regulator creates markets for these services in each location.

Using the theory developed in Section 7, a fully nonlinear problem can be also studied. In this case the linear or linearized dynamics used before for the resource stock can be replaced by the nonlinear dynamics of the form

$$
d x_{n}(t)=\left((\mathrm{A} x)_{n}+f\left(x_{n}\right)+(\Gamma u)_{n}+(\mathrm{C} v)_{n}\right) d t+(\mathrm{C} d w)_{n},
$$

where the operators $A, \Gamma, C$ have action such that

$$
(\mathrm{A} x)_{n}:=\sum_{m=0}^{N-1} \alpha_{n m} x_{m}(t),\left(\lceil u)_{n}:=\sum_{m=0}^{N-1} \gamma_{n m} u_{n m}(t),(\mathrm{C} v)_{n}:=\sum_{m=0}^{N-1} c_{n m} v_{m},\right.
$$

which corresponds to the total effect of the other sides stock, the total effect of the other sides control policies and the total effect of the other sides robustness policies at site $n$ respectively.

The regulators objective in this case, omitting the explicit dependence on $t$ to ease notation could take the more general form

$$
\min _{\left\{u_{n}(\cdot)\right\}} \max _{\left\{v_{n}(\cdot)\right\}} \mathbb{E}_{P}\left[\int_{0}^{\infty} e^{-r t} \sum_{n=0}^{N-1}\left[\sum_{m=0}^{N-1} U_{n m}^{1}\left(u_{n m}-b_{n m}\right)+U_{n}^{2}\left(x_{n}-\bar{x}_{n}\right)-\frac{\theta_{n}}{2}\left(v_{n}(t)\right)^{2}\right] d t\right]
$$

The linear operator A reflects the impact of the stock at different locations on the growth of the resource at location $n$. In this objective the regulator's utility is a function of (i) the deviation between consumption at location $m$ by individual $n$ and the corresponding bliss point, $U_{n m}^{1}$ (ii) the deviation between the stock of natural capital at location $n$ and some benchmark value $U_{n}^{2}$, and the penalty factor that reflects concerns regarding model misspecification.

As shown in Section 7, under the appropriate assumptions, this problem has a solution of the form

$$
u^{*}=D \mathrm{~K}^{£}\left(\mathrm{~B}^{*} D V(x)\right)
$$

where $V(x)$ is a solution of the Hamilton-Jacobi-Bellman-Isaacs equation. This solution will determine the equilibrium local prices by a condition similar to (51).

\footnotetext{
${ }^{22}$ The general case $b \neq 0$ is treated accordingly with minor modifications.
} 


\section{Hot spot interpretation}

The model presented in this section may for appropriate parameter values allow for the generation of hot spots of types I, II, III according to the general theory developed here. The following economic interpretation of these hot spots is possible:

$\triangleright$ Hot spot of type I: Regulation breaks down for small $\theta$. This means that because of the regulator has very strong concerns about possible model misspecifications at specific site(s) the regulator can not set up markets for consumption of in situ services where the supplied quantities satisfy the regulator's criterion.

$\triangleright$ Hot spot of type II: The regulator due to misspecification concerns allows a nonhomogeneous spatial pattern of the stocks to emerge. There exist a system of local prices that supports the spatial pattern.

$\triangleright$ Hot spot of type III: Due to misspecification concerns, the cost of controlling the in situ consumption at each location becomes very high in terms of deviations from the desired bliss points.

The parameter $\theta_{n}$ expressing misspecification concerns in site $n$ can, for certain problems, be related to the physical characteristics of the the site ${ }^{23}$. Thus if a hot spot is emerging from a given site this might signal the need for additional scientific evidence that might reduce the maximum misspecification error and thus the entropy constraint $H_{n}$. Reduction of the entropy constraint will increase $\theta_{n}$ and prevent the emergence of a hot spot.

\section{Concluding remarks}

We study robust control methods in a spatial domain where explicit spatial interactions are modelled by kernels and where concerns about model misspecification could be different across locations. We analyze linear quadratic problems. We derive closed form solutions for translation invariant systems but we also extent our results to general non translation invariant linear quadratic problems as well as to fully non linear systems. We show that misspecification concerns about specific cites could induce the emergence of hot spots which cause regulation to break down for the whole spatial domain. We also identify conditions for two more types of hot spots where location specific concerns could induce the emergence of spatial patters, or could render regulation very costly. We apply our methods to a problem of regulating in situ consumption when consumers are characterized by distance-dependent utility. We examine the emergence of local markets for in situ consumption and cases where location specific concerns could brake down regulation for the whole area, or could induce specific clustering.

Our results provide tools for studying optimal regulation of spatially interconnected systems when there are concerns about the specification of the model describing local processes describing the evolution of the system's states. Given the increasing interconnections and the localized uncertainties in the real world our approach could be appropriate for a wide class of economic problems characterized and connectivity, not necessarily spatial, since connectivity can be regarded with respect to other attributes, and by local uncertainties.

\section{References}

Akamatsu, T., Y. Takayama, and K. Ikeda (2009). Spatial discounting, fourier, and racetrack economy: A recipe for the analysis of spatial agglomeration models.

\footnotetext{
${ }^{23}$ See for example Athanassoglou and Xepapadeas (2012) for calibrating the parameter $\theta$ using scientific information related to climate change.
} 
Albeverio, S., K. Makarov, and A. Motovilov (2003). Graph subspaces and the spectral shift function. Canad. J. Math 55(3), 449-503.

Anderson, E., L. Hansen, and T. Sargent (2003). A quartet of semigroups for model specification, robustness, prices of risk, and model detection. Journal of the European Economic Association 1(1), 68-123.

Athanassoglou, S. and A. Xepapadeas (2012). Pollution control with uncertain stock dynamics: When, and how, to be precautious. Journal of Environmental Economics and Management 63, 304-320.

Aubin, J. and I. Ekeland (1984). Applied nonlinear analysis. Wiley New York.

Bamieh, B., F. Paganini, and M. Dahleh (2002). Distributed control of spatially invariant systems. Automatic Control, IEEE Transactions on 47(7), 1091-1107.

Başar, T. and P. Bernhard (2008). $H^{\infty}$-optimal control and related minimax design problems: a dynamic game approach. Birkhauser.

Bensoussan, A., G. Da Prato, M. Delfour, and S. Mitter (1992). Representation and Control of Infinite Dimensional Systems: Vol 2 (Systems $\mathcal{E}$ Control: Foundations $\&$ Applications). Birkhäuser Boston Inc., Boston, MA.

Boucekkine, R., C. Camacho, and B. Zou (2009). Bridging the gap between growth theory and the new economic geography: the spatial ramsey model. Macroeconomic Dynamics 13, 20-45.

Brock, W. and A. Xepapadeas (2008). Diffusion-induced instability and pattern formation in infinite horizon recursive optimal control. Journal of Economic Dynamics and Control 32(9), 2745-2787.

Brock, W. and A. Xepapadeas (2010). Pattern formation, spatial externalities and regulation in coupled economic-ecological systems. Journal of Environmental Economics and Management 59, 149-164.

Carmona, R. and M. Tehranchi (2006). Interest rate models: an infinite dimensional stochastic analysis perspective, Volume 13. Springer Verlag.

Cerrai, S. (2001). Second order PDE's in finite and infinite dimension: a probabilistic approach. Number 1762. Springer Verlag.

Curtain, R., O. Iftime, and H. Zwart (2008). System theoretic properties of platoon-type systems. In Decision and Control, 2008. CDC 2008. 47th IEEE Conference on, pp. 1442-1447. IEEE.

Da Prato, G. (2002). Linear quadratic control theory for infinite dimensional systems. Mathematical Control Theory. ICTP Lecture Notes 8, 59-105.

Da Prato, G. and J. Zabczyk (2002). Second order partial differential equations in Hilbert spaces, Volume 293. Cambridge Univ Pr.

Desmet, K. and E. Rossi-Hansberg (2010). On spatial dynamics*. Journal of Regional Science 50(1), 43-63.

Gilboa, I. and D. Schmeidler (1989). Maxmin expected utility with non-unique prior. Journal of Mathematical Economics 18, 141-153.

Haldane, A. (2009). Rethinking the financial network. Speech delivered at the Financial Student Association, Amsterdam, April. 
Hansen, L. and T. Sargent (2001). Robust control and model uncertainty. American Economic Review, $60-66$.

Hansen, L. and T. Sargent (2008). Robustness in Economic Dynamics. Princeton university press.

Hansen, L., T. Sargent, G. Turmuhambetova, and W. N. (2006). Robust control and model misspecification. Journal of Economic Theory 128, 45-90.

Isaacs, R. (1999). Differential games: a mathematical theory with applications to warfare and pursuit, control and optimization. Dover Pubns.

JET (2006). Symposium on model uncertainty and robustness. Journal of Economic Theory 128, $1-163$.

Karatzas, I. and S. Shreve (1991). Brownian motion and stochastic calculus. Springer.

Krugman, P. (1996). The Self-Organizing Economy. Blackwell.

Leizarowitz, A. (2008). Turnpike properties of a class of aquifer control problems. Automatica $44(6)$, $1460-1470$.

Ljungqvist, L. and T. Sargent (2004). Recursive macroeconomic theory. The MIT press.

Magill, M. (1977a). A local analysis of n-sector capital accumulation under uncertainty. Journal of Economic Theory 15(1), 211-219.

Magill, M. (1977b). Some new results on the local stability of the process of capital accumulation. Journal of Economic Theory 15(1), 174-210.

McMillan, C. and R. Triggiani (1994). Min-max game theory and algebraic riccati equations for boundary control problems with continuous input-solution map. part ii: The general case. Applied mathematics $\&$ optimization 29(1), 1-65.

Murray, J. (2003). Mathematical Biology, Vol. I and II (3rd Ed.). Springer.

Perrings, C. and B. Hannon (2001). An introduction to spatial discounting. Journal of Regional Science 41(1), 23-38.

Rudin, W. (1990). Fourier analysis on groups, Volume 12. Wiley-Interscience.

Salmon, M. (2002). Special issue on robust and risk sensitive decision theory. Macroeconomic Dynamics $6(1)$.

Sanchirico, J. and J. Wilen (1999). Bioeconomics of spatial exploitation in a patchy environment. Journal of Environmental Economics and Management 37(2), 129-150.

Smith, M., J. Sanchirico, and J. Wilen (2009). The economics of spatial-dynamic processes: applications to renewable resources. Journal of Environmental Economics and Management 57, 104-121.

Smith, T. (1975). An axiomatic theory of spatial discounting behavior. Papers in Regional Science 35(1), 31-44.

Smith, T. (1976). Spatial discounting and the gravity hypothesis. Regional Science and Urban Economics 6(4), 331-356.

Turing, A. (1952). The chemical basis of morphogenesis. Philosophical Transactions of the Royal Society of London 237, 37-72. 
Whittle, P. (1996). Optimal control: basics and beyond, Volume 18. John Wiley \& Sons.

Wilen, J. (2007). Economics of spatial-dynamic processes. American Journal of Agricultural Economics 89(5), 1134-1144.

Wong, M. (2011). Discrete Fourier Analysis, Volume 6. Birkhauser.

Wu, J. and A. Plantinga (2003). The influence of public open space on urban spatial structure. Journal of Environmental Economics and Management 46(2), 288-309. 